\title{
Macroscopic behavior of materials composed of two elastic media
}

\author{
Harald Pleiner $\odot,{ }^{1, *}$ Andreas M. Menzel $\odot,{ }^{2, \dagger}$ and Helmut R. Brand $\odot^{3,1, \dagger}$ \\ ${ }^{1}$ Max Planck Institute for Polymer Research, 55021 Mainz, Germany \\ ${ }^{2}$ Institut für Physik, Otto-von-Guericke-Universität Magdeburg, Universitätsplatz 2, 39106 Magdeburg, Germany \\ ${ }^{3}$ Department of Physics, University of Bayreuth, 95440 Bayreuth, Germany
}

(Received 11 January 2021; revised 2 May 2021; accepted 6 May 2021; published 18 May 2021)

\begin{abstract}
In the present paper we investigate macroscopic two-fluid effects in systems composed of two elastic media. Using two strain tensors as macroscopic variables, we present a nonlinear analysis in the regime of long wavelengths and low frequencies. We also discuss the description in terms of relative strains and total strains. Generalizing earlier work on relative rotations for systems such as nematic elastomers or uniaxial magnetic gels, we investigate how this concept can be applied to the case of two elastic media in the linear domain. For the two strain fields and relative rotations between the two elastic media, we find a number of reversible and dissipative cross-coupling terms that couple velocity differences, mean velocities, strain fields, and relative rotations to each other as well as to temperature and concentration gradients. The question of relative translations is also analyzed. A linearized description using relative translations is physically meaningful as well as technically consistent with using strain fields and relative rotations. Finally, we apply this description to the swinging of two coupled homogeneous elastic media relative to each other, and to the oscillating actuation or active stress introduced through one of the elastic compounds.
\end{abstract}

DOI: 10.1103/PhysRevB.103.174304

\section{INTRODUCTION}

There are many elastic and viscoelastic composite materials ranging from interpenetrating hydrogel polymer networks [1] over fiber networks embedded in a matrix [2,3], microtubuli coupled to the elastic cytoskeleton in cells [4], polymeric materials reinforced by carbon nanotubes [5], block copolymers [6,7], to concrete (see Ref. [8], and references therein). A large fraction of the studies of such composite materials is addressing the optimization of static elastic properties by varying the concentration of the various components, the rigidity, and the particle size. References [1-10] give a cross section of these efforts to characterize, by several different techniques, the static elastic and also viscoelastic properties of such multicomponent materials.

Several of the individual constituents of the mentioned viscoelastic composite systems are anisotropic; for instance, fibers or microtubuli. However, they may be arranged in a disordered state so that their macroscopic response appears isotropic. In the present paper, we restrict our analysis to isotropic materials. For example, it is well known how to

\footnotetext{
*pleiner@mpip-mainz.mpg.de

†a.menzel@ovgu.de

*brand@uni-bayreuth.de
}

Published by the American Physical Society under the terms of the Creative Commons Attribution 4.0 International license. Further distribution of this work must maintain attribution to the author(s) and the published article's title, journal citation, and DOI. Open access publication funded by the Max Planck Society. generalize material laws of elasticity for isotropic solids to anisotropic solids; compare, e.g., Ref. [11] for linear crystal elasticity. We emphasize that the structure of the underlying constitutive equations is unchanged by considering anisotropic instead of isotropic materials, while the number of independent coefficients in the associated property tensors will vary significantly. As for linear elasticity, we have two independent elastic coefficients for isotropic materials but 21 coefficients for triclinic substances.

This situation has further developed in recent years, when in systems that can be controlled by external fields, such as magnetic fields in the case of magnetic elastomers [12,13], particularly the dynamic and viscoelastic properties as well as the dynamics of the patterns formed inside the materials are increasingly investigated. In addition to traditional rigid compound solids, the study of functionalized compound materials emerges. For example, an individual component in such multicomponent systems may already constitute a functionalized material by itself, e.g., a liquid crystalline gel or an elastomer functionalized by magnetic inclusions.

A general framework to derive the macroscopic dynamic equations for systems with two immiscible subsystems has been set up in Refs. [14,15]. In these two references a first set of applications to immiscible fluid mixtures, a two-fluid system for which one fluid is nematic and a two-fluid system with one strain field characteristic for a solid has been studied as well $[14,15]$. In the field of bioinspired nonequilibrium systems the polar dynamic preferred direction emerges as a second velocity field. This feature has been exploited to derive the active polar two-fluid macroscopic dynamics for such a system [16]. The approach has been generalized to incorporate a viscoelastic background [17] thus also 
addressing transient and/or permanent networks. Quite recently [18] the macroscopic approach has been applied to two-fluid effects in magnetorheological fluids, thus demonstrating its applicability to device-related questions such as the tunability of a material by moderate external magnetic fields. We here generalize the approach to two-component materials that both may feature elastic properties, thus extending a previous macroscopic two-component description [8] to the two-fluid dynamical regime.

Historically the best characterized and studied two-fluid systems are superfluids, namely, superfluid ${ }^{4} \mathrm{He}$ and the superfluid phases of ${ }^{3} \mathrm{He}$ [19-25]. However, in those quantum systems, the second (or superfluid) velocity is due to the spontaneously broken gauge symmetry, and is therefore a truly hydrodynamic degree of freedom. This situation must be contrasted with two-fluid effects in all the other systems discussed above and with the systems to be examined in the following that are not superfluid. In this case only the barycentric velocity is a truly hydrodynamic variable and has no gap in the excitation spectrum in the long wavelength limit. In contrast, the relative velocity, although long lived and therefore kept on the list of macroscopic variables, acquires a gap for small wave vectors.

In systems composed of two subsystems, which can be rotated relative to each other, relative rotations can emerge as macroscopic variables. As examples of such materials, we mention relative rotations in liquid crystalline elastomers as pioneered by de Gennes [26] and uniaxial magnetic gels [27]. Quite recently it has become clear that relative rotations are also of interest in ferromagnetic gel phases with tetrahedral order [28]. For a generalization of the concept of relative rotations to the nonlinear domain we refer to Refs. [29-31].

Finally we also address the question of relative translations for the type of solid systems studied here, which could come as macroscopic variables, in general, in addition to the two strain fields and the relative rotations mentioned already. It turns out that only linearized relative translations are physically sensible in order to obtain a consistent picture.

In classical elastostatics the focus is typically on the Lagrange version of the strain tensor. In contrast we will use the Euler description throughout, because we also want to incorporate dynamic constitutive equations or material laws. Various detailed aspects of this description have been given in Refs. [32-38]. In case one is interested to study nonlinear effects for large strains, we refer to Refs. [39,40], where this has been analyzed in the framework of transient nonlinear elasticity.

The paper is organized as follows. The bulk is dedicated to the study of the nonlinear macroscopic behavior of systems with two strain fields. Their description is given in Secs. II (macroscopic variables), III (statics), IV (dynamics), V (dissipative currents), and VI (reversible currents). In Sec. VII we discuss how relative rotations enter the picture, and in Sec. VIII we analyze the incorporation of relative translations. Two basic minimal example situations are considered in Sec. IX. They illustrate the importance of the central aspect in the present work, namely, including separate macroscopic velocity variables for both components in elastic composite materials. We summarize our work while providing conclusions and perspectives in Sec. X. In Appendix A we demonstrate how the complexity of the dynamic strain equations may be reduced by different types of approximations, and in Appendix B we recall some general details of two-fluid hydrodynamics.

\section{VARIABLES}

The hydrodynamics of an elastic continuum is described by the momentum density $g_{i}$, the mass density $\rho$, and the total energy density $\varepsilon$ representing the local conservation laws of a fluid, as well as by the strain tensor $U_{i j}$. The latter is symmetric and quantifies elastic deformations. Often, elasticity is described using a field $u_{i}$ denoting the displacement of any point of the continuum. For a crystal $u_{i}$ is the symmetry variable of the spontaneously broken translational symmetry. Since it contains also spatially homogeneous translations and homogeneous rotations that both do not lead to elastic deformations, one takes symmetrized gradients of $u_{i}$, in particular $U_{i j}=(1 / 2)\left(\nabla_{i} u_{j}+\nabla_{j} u_{i}-\left[\nabla_{k} u_{i}\right]\left[\nabla_{k} u_{j}\right]\right)$. Note that hydrodynamics uses the Eulerian picture, where all variables are space-time fields in the (usually Cartesian) laboratory space. In contrast, in elastostatics often the Lagrangian picture is used, where elastic deformations are described in the frame of the deformed body (because no other hydrodynamic variables are present). This immediately leads to the observation that $u_{i}$ is not suitable as a variable in (nonlinear) hydrodynamics, since it is interpreted differently in the Eulerian and Lagrangian picture. Fortunately, it is possible to define and introduce $U_{i j}$ without making any reference to $u_{i}$. For a thorough discussion of these points, see Refs. [32-34].

In a system composed of two elastic media, the elastic properties are described by two strain fields $U_{i j}^{(1)}$ and $U_{i j}^{(2)}$. Likewise, we can use the linear combinations, total strain, $2 U_{i j}^{(\text {tot })}=U_{i j}^{(1)}+U_{i j}^{(2)}$, and relative strain [8], $2 U_{i j}^{(\text {rel })}=U_{i j}^{(1)}-$ $U_{i j}^{(2)}$. There are two mass densities $\rho^{(n)}$ and two velocities $v_{i}^{(n)}$ giving rise to two momentum densities $g_{i}^{(n)}=\rho^{(n)} v_{i}^{(n)}$ with $n \in\{1,2\}$. Often, one uses as variables the total mass density $\rho \equiv \rho^{(1)}+\rho^{(2)}$ and a concentration variable $\phi \equiv \rho^{(1)} / \rho$, as well as the total momentum density $g_{i} \equiv g_{i}^{(1)}+g_{i}^{(2)}$ and the relative velocity $w_{i} \equiv v_{i}^{(1)}-v_{i}^{(2)}$. For details of these twofluid aspects, see Ref. [14].

In a system composed of two elastic media, a simultaneous and identical rigid translation of both subsystems does not change the internal state and is therefore not included as a variable in our description (as in the single-elastic case). However, relative translations between the two subsystems, $u_{i}^{(\mathrm{rel})} \equiv u_{i}^{(1)}-u_{i}^{(2)}$, are variables to be included, describing, e.g., homogeneous relative translations. We will discuss this type of variable, and its couplings to the others, in Sec. VIII separately.

In addition, in a system composed of two elastic media, a rigid combined simultaneous rotation of both subsystems is equivalent to a rotation of the inertial system and must not change the internal state of the media (as in the singleelastic case). Relative rotations between the two subsystems, however, are macroscopic variables. They are not related to any conservation law or broken symmetry, but may relax in a finite time, if the two subsystems are coupled to each other. Therefore, they are relevant, if their relaxation times are in 
the time range of the other (hydrodynamic) variables. We will deal with the dynamics of relative rotations in Sec. VII separately.

The first law of thermodynamics relates changes of the variables to changes of the energy density $\varepsilon$ by the Gibbs relation $[41,42]$

$$
\begin{aligned}
d \varepsilon= & T d \sigma+\mu d \rho+\Pi d \phi+\boldsymbol{v} \cdot d \boldsymbol{g}+\boldsymbol{m} \cdot d \boldsymbol{w} \\
& +\Phi_{i j}^{(1)} d U_{i j}^{(1)}+\Phi_{i j}^{(2)} d U_{i j}^{(2)},
\end{aligned}
$$

where we can likewise replace $\Phi_{i j}^{(1)} d U_{i j}^{(1)}+\Phi_{i j}^{(2)} d U_{i j}^{(2)}$ by $\Phi_{i j}^{(\text {tot })} d U_{i j}^{(\text {tot })}+\Phi_{i j}^{(\text {rel) }} d U_{i j}^{(\text {rel })}$. The entropy density $\sigma$ represents the thermal degree of freedom of the system. The appropriate thermodynamic conjugates are the temperature $T$, the chemical potential $\mu$, the osmotic pressure (times the density $\rho$ ) $\Pi$, the mean velocity $v_{i}=g_{i} / \rho$, the conjugate field $m_{i}$, and the two elastic stress fields, $\Phi_{i j}^{(1)}$ and $\Phi_{i j}^{(2)}$, or likewise the total elastic stress field $2 \Phi_{i j}^{(\text {tot })}=\Phi_{i j}^{(1)}+\Phi_{i j}^{(2)}$ and the relative elastic stress field $2 \Phi_{i j}^{(\text {rel })}=\Phi_{i j}^{(1)}-\Phi_{i j}^{(2)}$. The corresponding framework for pure two-component fluids in the absence of elasticity is briefly reviewed in Appendix B.

Rotational invariance of the Gibbs relation (1) including elasticity leads to the condition

$$
U_{i k}^{(1)} \Phi_{k j}^{(1)}+U_{i k}^{(2)} \Phi_{k j}^{(2)}=U_{j k}^{(1)} \Phi_{k i}^{(1)}+U_{j k}^{(2)} \Phi_{k i}^{(2)}
$$

From the requirement that the energy of the system is a firstorder Eulerian form of all extensive variables, one gets for the pressure $p \equiv-(\partial / \partial V) \int \varepsilon d V$ the Gibbs-Duhem relation

$$
\begin{aligned}
d p= & \sigma d T+\rho d \mu-\Pi d \phi+\boldsymbol{g} \cdot d \boldsymbol{v} \\
& -\boldsymbol{m} \cdot d \boldsymbol{w}-\Phi_{i j}^{(1)} d U_{i j}^{(1)}-\Phi_{i j}^{(2)} d U_{i j}^{(2)} .
\end{aligned}
$$

\section{STATICS}

The static behavior of our macroscopic system is conveniently described by the energy functional

$$
\begin{aligned}
\varepsilon & =\frac{T}{2 C_{V}}(\delta \sigma)^{2}+\frac{1}{2 \kappa_{\phi}}(\delta \phi)^{2}+\frac{1}{2 \rho^{2} \kappa_{\mu}}(\delta \rho)^{2}+\frac{1}{\alpha_{\phi}}(\delta \phi)(\delta \sigma)+\frac{1}{\rho \alpha_{\rho}}(\delta \rho)(\delta \sigma)+\frac{1}{\rho \kappa_{\pi}}(\delta \rho)(\delta \phi) \\
& +\frac{1}{2} c_{t r}^{(11)} \tilde{U}_{i j}^{(1)} \tilde{U}_{i j}^{(1)}+\frac{1}{2} c_{t r}^{(22)} \tilde{U}_{i j}^{(2)} \tilde{U}_{i j}^{(2)}+c_{t r}^{(12)} \tilde{U}_{i j}^{(1)} \tilde{U}_{i j}^{(2)}+\frac{1}{2} c_{l}^{(11)} U_{k k}^{(1)} U_{l l}^{(1)}+\frac{1}{2} c_{l}^{(22)} U_{k k}^{(2)} U_{l l}^{(2)}+c_{l}^{(12)} U_{k k}^{(1)} U_{l l}^{(2)} \\
& +\frac{1}{\alpha_{1}} U_{k k}^{(1)} \delta \sigma+\frac{1}{\alpha_{2}} U_{k k}^{(2)} \delta \sigma+\frac{1}{\rho \kappa_{u 1}} U_{k k}^{(1)} \delta \phi+\frac{1}{\rho \kappa_{u 2}} U_{k k}^{(2)} \delta \phi+\frac{1}{\rho \kappa_{\rho 1}} U_{k k}^{(1)} \delta \rho+\frac{1}{\rho \kappa_{\rho 2}} U_{k k}^{(2)} \delta \rho,
\end{aligned}
$$

from which the conjugate fields follow by partial derivation according to the Gibbs relation, Eq. (1),

$$
\begin{gathered}
\delta T=\frac{T}{C_{V}} \delta \sigma+\frac{1}{\alpha_{\phi}} \delta \phi+\frac{1}{\rho \alpha_{\rho}} \delta \rho+\frac{1}{\alpha_{1}} U_{k k}^{(1)}+\frac{1}{\alpha_{2}} U_{k k}^{(2)}, \\
\Pi=\frac{1}{\kappa_{\phi}} \delta \phi+\frac{1}{\rho \kappa_{\pi}} \delta \rho+\frac{1}{\alpha_{\phi}} \delta \sigma+\frac{1}{\rho \kappa_{u 1}} U_{k k}^{(1)}+\frac{1}{\rho \kappa_{u 2}} U_{k k}^{(2)}+\boldsymbol{w} \cdot \boldsymbol{g}+\rho \boldsymbol{w}^{2}(1-2 \phi), \\
\mu=\frac{1}{\rho^{2} \kappa_{\mu}} \delta \rho+\frac{1}{\rho \kappa_{\pi}} \delta \phi+\frac{1}{\rho \alpha_{\rho}} \delta \sigma+\frac{1}{\rho \kappa_{\rho 1}} U_{k k}^{(1)}+\frac{1}{\rho \kappa_{\rho 2}} U_{k k}^{(2)}+\boldsymbol{w}^{2} \phi(1-\phi), \\
\Phi_{k k}^{(1)}=c_{l}^{(11)} U_{k k}^{(1)}+c_{l}^{(12)} U_{k k}^{(2)}+\frac{1}{\alpha_{1}} \delta \sigma+\frac{1}{\rho \kappa_{u 1}} \delta \phi+\frac{1}{\rho \kappa_{\rho 1}} \delta \rho, \\
\Phi_{k k}^{(2)}=c_{l}^{(22)} U_{k k}^{(2)}+c_{l}^{(12)} U_{k k}^{(1)}+\frac{1}{\alpha_{2}} \delta \sigma+\frac{1}{\rho \kappa_{u 2}} \delta \phi+\frac{1}{\rho \kappa_{\rho 2}} \delta \rho, \\
\tilde{\Phi}_{i j}^{(1)}=c_{t r}^{(11)} \tilde{U}_{i j}^{(1)}+c_{t r}^{(12)} \tilde{U}_{i j}^{(2)}, \\
\tilde{\Phi}_{i j}^{(2)}=c_{t r}^{(22)} \tilde{U}_{i j}^{(2)}+c_{t r}^{(12)} \tilde{U}_{i j}^{(1)} .
\end{gathered}
$$

We have split the strain fields and the elastic stresses into traces, $U_{k k}^{(1,2)}, \Phi_{k k}^{(1,2)}$, and traceless parts, $\tilde{U}_{i j}^{(1,2)}=U_{i j}^{(1,2)}-$ $(1 / 3) \delta_{i j} U_{k k}^{(1,2)}, \quad \tilde{\Phi}_{i j}^{(1,2)}=\Phi_{i j}^{(1,2)}-(1 / 3) \delta_{i j} \Phi_{k k}^{(1,2)}$, which is common in isotropic linear elasticity. There are six static susceptibilities from the binary mixture fluid $\left(C_{V}, \alpha_{\phi}, \alpha_{\rho}, \kappa_{\phi}\right.$, $\kappa_{\pi}$, and $\kappa_{\mu}$ ), six elastic Hooke-like moduli from the two elastic media (longitudinal $c_{l}^{(11,12,22)}$ and transverse $c_{t r}^{(11,12,22)}$ ), and six general susceptibilities describing the cross-coupling between the fluid and the elastic degrees of freedom $\left(\alpha_{1}, \alpha_{2}\right.$, $\kappa_{u 1}, \kappa_{u 2}, \kappa_{\rho 1}$, and $\left.\kappa_{\rho 2}\right)$. In linear approximation there is one coefficient related to relative translations and relative rotations (cf., respectively, Secs. VII and VIII below), and there are no static cross-couplings to other variables. It should be noted that for real solids at finite temperatures $U_{k k}^{(n)} \neq \delta \rho^{(n)} / \rho$ in contrast to ideal elasticity theory. The reason is the point defects, which allow not only the dissipative motion described below, but also static temperature and pressure changes due to $U_{k k}^{(n)}$ even at constant density.

The set of static relations, Eqs. (5)-(11), includes linear elasticity described by bilinear expressions of the strains in the energy functional. A generalization to nonlinear elasticity is conveniently achieved, e.g., by adding appropriate nonlinear expressions beyond the harmonic approximation to the energy functional (cf. Refs. [39,40]). The elastic coefficients of each component of a composite material can vary by many orders of magnitude. For example, the elastic Young's modulus, $E$, ranges from several hundred GPa for metals like osmium [43] 
down to values of around $1 \mathrm{~Pa}$ for soft gels [44], which constitutes as much as 11 orders of magnitude. There is also a rich literature on how to incorporate higher-order elastic effects such as third-order elasticity into a macroscopic description. Compare, for example, Refs. [32,34] for general aspects and Refs. $[39,40]$ for polymeric-type materials. As for the pressure dependence of static susceptibilities, such as elastic constants, we have expanded the generalized energy density $\varepsilon$, Eq. (4), up to second order in the changes of the macroscopic variables entropy density $\delta \sigma$, density $\delta \rho$, concentration $\delta \phi$, and in the strain tensors $U_{i j}^{(1,2)}$. Thus, the expansion coefficients, e.g., the elastic moduli, here are assumed to be constant. To obtain, for instance, the pressure dependence of the elastic coefficients, one has to calculate their derivatives with respect to pressure, ideally involving the appropriate equation of state.

Finally, the remaining relations between conjugates and variables

$$
v_{i}=\frac{g_{i}}{\rho} \quad \text { and } \quad m_{i}=\phi(1-\phi) \rho w_{i} \equiv \alpha w_{i}
$$

are not really static, but nevertheless follow from the energy density, in particular from the kinetic energy density $\varepsilon_{\text {kin }}=$ $\left(1 / 2 \rho^{(1)}\right)\left[\boldsymbol{g}^{(1)}\right]^{2}+\left(1 / 2 \rho^{(2)}\right)\left[\boldsymbol{g}^{(2)}\right]^{2}=(1 / 2) \boldsymbol{g}^{2}+(\alpha / 2) \boldsymbol{w}^{2}$. The $w_{i}$ dependence of the chemical potential and the osmotic pressure are due to the $\rho$ and $\phi$ dependence of $\alpha$.

\section{DYNAMICS}

The dynamic equations for the elastomeric and fluid degrees of freedom are

$$
\begin{gathered}
\dot{\epsilon}+\nabla_{i}(\epsilon+p) v_{i}+\nabla_{i}\left(j_{i}^{(\epsilon) R}+j_{i}^{(\epsilon) D}\right)=0, \\
\dot{\sigma}+\nabla_{i}\left(\sigma v_{i}+j_{i}^{(\sigma) R}+j_{i}^{(\sigma) D}\right)=2 R / T \\
\dot{\rho}+\nabla_{j} \rho v_{j}=0 \\
\dot{\phi}+v_{j} \nabla_{j} \phi+\rho^{-1} \nabla_{i}\left[\rho \phi(1-\phi) w_{i}+j_{i}^{(1) R}+j_{i}^{(1) D}\right]=0, \\
\dot{g}_{i}+\nabla_{j} g_{i} v_{j}+\nabla_{i} p+\nabla_{j}\left(-\Phi_{i j}^{(\mathrm{tot})}+\sigma_{i j}^{(\mathrm{conv})}+\sigma_{i j}^{R}+\sigma_{i j}^{D}\right)=0, \\
\dot{w}_{i}+v_{j} \nabla_{j} w_{i}+\nabla_{i}\left(\rho^{-1} \Pi\right)+X_{i}^{R}+X_{i}^{D}=0, \\
\dot{U}_{i j}^{(1)}+v_{k} \nabla_{k} U_{i j}^{(1)}+U_{k j}^{(1)} \nabla_{i} v_{k}+U_{k i}^{(1)} \nabla_{j} v_{k}-A_{i j}+Z_{i j}^{(1) R}+Z_{i j}^{(1) D}=0, \\
\dot{U}_{i j}^{(2)}+v_{k} \nabla_{k} U_{i j}^{(2)}+U_{k j}^{(2)} \nabla_{i} v_{k}+U_{k i}^{(2)} \nabla_{j} v_{k}-A_{i j}+Z_{i j}^{(2) R}+Z_{i j}^{(2) D}=0
\end{gathered}
$$

with $2 A_{i j}=\nabla_{i} v_{j}+\nabla_{j} v_{i}$, and $R$ the energy dissipation function. The elastic convective stress is

$$
\sigma_{i j}^{(\mathrm{conv})}=\Phi_{j k}^{(1)} U_{i k}^{(1)}+\Phi_{i k}^{(1)} U_{j k}^{(1)}+\Phi_{j k}^{(2)} U_{i k}^{(2)}+\Phi_{i k}^{(2)} U_{j k}^{(2)}=\Phi_{j k}^{(\mathrm{tot})} U_{i k}^{(\mathrm{tot})}+\Phi_{i k}^{(\mathrm{tot})} U_{j k}^{(\mathrm{tot})}+\Phi_{j k}^{(\mathrm{rel})} U_{i k}^{(\mathrm{rel})}+\Phi_{i k}^{(\mathrm{rel})} U_{j k}^{(\mathrm{rel})} .
$$

These equations follow from Refs. [14,32,33] and contain, apart from the reversible (superscript $R$ ) and irreversible, dissipative (superscript $D$ ) phenomenological currents, also transport, convection, and translation whenever appropriate. The latter are reversible and, indeed, all transport contributions (including the isotropic pressure) add up to zero entropy production. The convective parts in the strain equations are compensated by $\sigma_{i j}^{(\text {conv })}$, and the translational term $\left(A_{i j}\right)$ gives rise to the elastic stresses. Instead of Eqs. (19) and (20) one can likewise use

$$
\begin{array}{r}
\dot{U}_{i j}^{(\mathrm{tot})}+v_{k} \nabla_{k} U_{i j}^{(\mathrm{tot})}+U_{k j}^{(\mathrm{tot})} \nabla_{i} v_{k}+U_{k i}^{(\mathrm{tot})} \nabla_{j} v_{k}-A_{i j}+Z_{i j}^{(\mathrm{tot}) \mathrm{R}}+Z_{i j}^{(\mathrm{tot}) \mathrm{D}}=0 \\
\dot{U}_{i j}^{(\mathrm{rel})}+v_{k} \nabla_{k} U_{i j}^{(\mathrm{rel})}+U_{k j}^{(\mathrm{rel})} \nabla_{i} v_{k}+U_{k i}^{(\mathrm{rel})} \nabla_{j} v_{k}+Z_{i j}^{(\mathrm{rel}) \mathrm{R}}+Z_{i j}^{(\mathrm{rel}) \mathrm{D}}=0
\end{array}
$$

where $2 Z_{i j}^{(\text {tot }) *}=Z_{i j}^{(1) *}+Z_{i j}^{(2) *}$ and $2 Z_{i j}^{(\text {rel }) *}=Z_{i j}^{(1) *}-Z_{i j}^{(2) *}$, for $* \in\{R, D\}$.

In the whole set of dynamic equations the mean velocity $v_{i}$ has been chosen as the convective velocity for all variables. This ensures zero entropy production of the convective derivatives including the corotational and the translational parts. Due to various material dependent contributions in the reversible currents (see below), the actual convective velocities can be different from $v_{i}$ and can be specific for the different variables.

For the phenomenological parts of the currents the second law of thermodynamics requires

$$
R=-j_{i}^{(\sigma) *} \nabla_{i} T-j_{i}^{(1) *} \nabla_{i}(\Pi / \rho)-\sigma_{i j}^{*} \nabla_{j} v_{i}+\Phi_{i j}^{(1)} Z_{i j}^{(1) *}+\Phi_{i j}^{(2)} Z_{i j}^{(2) *}+m_{i} X_{i}^{*} \geqslant 0
$$

with the equal sign $(>\operatorname{sign})$ for $*=R(*=D)$. 


\section{DISSIPATIVE CURRENTS}

The dissipative parts of the currents introduced above can be deduced from a potential, the dissipation function $R$, that reads in bilinear approximation

$$
\begin{aligned}
2 R= & \kappa(\nabla T)^{2}+D(\nabla \Pi)^{2}+2 D^{(T)}(\nabla T) \cdot(\nabla \Pi)+\xi^{\prime} m^{2}+\zeta_{i j k l}^{(11)} \Phi_{i j}^{(1)} \Phi_{k l}^{(1)}+\zeta_{i j k l}^{(22)} \Phi_{i j}^{(2)} \Phi_{k l}^{(2)}+2 \zeta_{i j k l}^{(12)} \Phi_{i j}^{(1)} \Phi_{k l}^{(2)} \\
& +v_{i j k l}\left(\nabla_{j} v_{i}\right)\left(\nabla_{l} v_{k}\right)+2 v_{i j k l}^{(c)}\left(\nabla_{j} v_{i}\right)\left(\nabla_{l} m_{k}\right)+v_{i j k l}^{(w)}\left(\nabla_{j} m_{i}\right)\left(\nabla_{l} m_{k}\right) \\
& +\xi_{l}^{(11)}\left(\nabla_{k} \Phi_{i k}^{(1)}\right)\left(\nabla_{l} \Phi_{i l}^{(1)}\right)+\xi_{l}^{(22)}\left(\nabla_{k} \Phi_{i k}^{(2)}\right)\left(\nabla_{l} \Phi_{i l}^{(2)}\right)+2 \xi_{l}^{(12)}\left(\nabla_{k} \Phi_{i k}^{(1)}\right)\left(\nabla_{l} \Phi_{i l}^{(2)}\right) \\
& +\xi_{t r}^{(11)}\left(\nabla_{i} \Phi_{k k}^{(1)}\right)\left(\nabla_{i} \Phi_{l l}^{(1)}\right)+\xi_{t r}^{(22)}\left(\nabla_{i} \Phi_{k k}^{(2)}\right)\left(\nabla_{i} \Phi_{l l}^{(2)}\right)+2 \xi_{t r}^{(12)}\left(\nabla_{i} \Phi_{k k}^{(1)}\right)\left(\nabla_{i} \Phi_{l l}^{(2)}\right) \\
& +2 \kappa_{1}\left(\nabla_{i} \Phi_{k k}^{(1)}\right)\left(\nabla_{i} T\right)+2 \kappa_{2}\left(\nabla_{i} \Phi_{k k}^{(2)}\right)\left(\nabla_{i} T\right)+2 d_{1}\left(\nabla_{i} \Phi_{k k}^{(1)}\right)\left(\nabla_{i} \Pi\right)+2 d_{2}\left(\nabla_{i} \Phi_{k k}^{(2)}\right)\left(\nabla_{i} \Pi\right),
\end{aligned}
$$

where all four-rank material tensors have the form $v_{i j k l}=v_{l} \delta_{i j} \delta_{k l}+v_{t r}\left[\delta_{i k} \delta_{j l}+\delta_{i l} \delta_{j k}-(2 / 3) \delta_{i j} \delta_{k l}\right] / 2$ and contain two parameters each. The parameters $\zeta_{l}$ and $\zeta_{t r}$ describe the relaxation of elastic strains in the case of nonpermanent elastic media. Conversely, the parameters $\xi_{l, t r}$ quantify diffusion that takes place even in permanent elastic media due to vacancy diffusion. In elasticity theory very often the latter is neglected and the dynamics of strains is considered to be purely reversible. Concerning the diagonal elements of $R$, which quantify diffusion or relaxation of a single variable, all diagonal coefficients must be positive, in particular $\kappa, D, \xi^{\prime}, \zeta_{l}^{(11)}, \zeta_{l}^{(22)}, \zeta_{t r}^{(11)}, \zeta_{t r}^{(22)}, v_{l}, v_{t r}, v_{l}^{(w)}, v_{t r}^{(w)}, \xi_{t r}^{(11)}, \xi_{t r}^{(22)}, \xi_{l}^{(11)}, \xi_{l}^{(22)}$. Moreover, the cross-coupling coefficients are bound in magnitude to guarantee $R>0$ and have to obey

$$
\begin{aligned}
& \left(D^{(T)}\right)^{2}<\kappa D, \quad\left(\zeta_{l}^{(12)}\right)^{2}<\zeta_{l}^{(11)} \zeta_{l}^{(22)}, \quad\left(\zeta_{t r}^{(12)}\right)^{2}<\zeta_{t r}^{(11)} \zeta_{t r}^{(22)}, \quad\left(v_{l}^{(c)}\right)^{2}<v_{l} v_{l}^{(w)}, \quad\left(v_{t r}^{(c)}\right)^{2}<v_{t r} v_{t r}^{(w)}, \\
& \left(\xi_{t r}^{(12)}\right)^{2}<\xi_{t r}^{(11)} \xi_{t r}^{(22)}, \quad\left(\xi_{l}^{(12)}\right)^{2}<\xi_{l}^{(11)} \xi_{l}^{(22)}, \quad \kappa_{1}^{2}<\kappa \xi_{t r}^{(11)}, \quad \kappa_{2}^{2}<\kappa \xi_{t r}^{(22)}, \quad d_{1}^{2}<D \xi_{t r}^{(11)}, \quad d_{2}^{2}<D \xi_{t r}^{(22)} .
\end{aligned}
$$

From Eq. (25) the following dissipative currents are obtained:

$$
\begin{aligned}
& j_{i}^{(\sigma) D}=-(\partial R) /\left(\partial \nabla_{i} T\right)=-\kappa \nabla_{i} T-\phi(1-\phi) d^{(T)} \nabla_{i} \Pi-\kappa_{1} \nabla_{i} \Phi_{k k}^{(1)}-\kappa_{2} \nabla_{i} \Phi_{k k}^{(2)}, \\
& Z_{i j}^{(1) D}=(\delta R) /\left(\delta \Phi_{i j}^{(1)}\right)=\zeta_{l}^{(11)} \delta_{i j} \Phi_{k k}^{(1)}+\zeta_{l}^{(12)} \delta_{i j} \Phi_{k k}^{(2)}+\zeta_{t r}^{(11)} \tilde{\Phi}_{i j}^{(1)}+\zeta_{t r}^{(12)} \tilde{\Phi}_{i j}^{(2)} \\
&-\frac{1}{2} \nabla_{j}\left(\xi_{t r}^{(11)} \nabla_{k} \Phi_{i k}^{(1)}+\xi_{t r}^{(12)} \nabla_{k} \Phi_{i k}^{(2)}\right)-\frac{1}{2} \nabla_{i}\left(\xi_{t r}^{(11)} \nabla_{k} \Phi_{j k}^{(1)}+\xi_{t r}^{(12)} \nabla_{k} \Phi_{j k}^{(2)}\right) \\
&-\delta_{i j} \nabla_{k}\left(\xi_{l}^{(11)} \nabla_{k} \Phi_{l l}^{(1)}+\xi_{l}^{(12)} \nabla_{k} \Phi_{l l}^{(2)}\right)-\kappa_{1} \delta_{i j} \nabla^{2} T-d_{1} \delta_{i j} \nabla^{2} \Pi, \\
& Z_{i j}^{(2) D}=(\delta R) /\left(\delta \Phi_{i j}^{(2)}\right)=\zeta_{l}^{(22)} \delta_{i j} \Phi_{k k}^{(2)}+\zeta_{l}^{(12)} \delta_{i j} \Phi_{k k}^{(1)}+\zeta_{t r}^{(22)} \tilde{\Phi}_{i j}^{(2)}+\zeta_{t r}^{(12)} \tilde{\Phi}_{i j}^{(1)} \\
&-\frac{1}{2} \nabla_{j}\left(\xi_{t r}^{(22)} \nabla_{k} \Phi_{i k}^{(2)}+\xi_{t r}^{(12)} \nabla_{k} \Phi_{i k}^{(1)}\right)-\frac{1}{2} \nabla_{i}\left(\xi_{t r}^{(2)} \nabla_{k} \Phi_{j k}^{(2)}+\xi_{t r}^{(12)} \nabla_{k} \Phi_{j k}^{(1)}\right) \\
&-\delta_{i j} \nabla_{k}\left(\xi_{l}^{(22)} \nabla_{k} \Phi_{l l}^{(2)}+\xi_{l}^{(12)} \nabla_{k} \Phi_{l l}^{(1)}\right)-\kappa_{2} \delta_{i j} \nabla^{2} T-d_{2} \delta_{i j} \nabla^{2} \Pi, \\
& \sigma_{i j}^{D}=-(\partial R) /\left(\partial \nabla_{j} v_{i}\right)=-v_{i j k l} \nabla_{l} v_{k}-v_{i j k l}^{(c)} \nabla_{l} m_{k}, \\
& X_{i}^{D}=(\delta R) /\left(\delta m_{i}\right)=\xi^{\prime} m_{i}-\nabla_{j}\left(v_{i j k l}^{(w)} \nabla_{l} m_{k}+v_{k l i j}^{(c)} \nabla_{l} v_{k}\right), \\
& j_{i}^{(1) D}=-\rho(\partial R) /\left(\partial \nabla_{i} \Pi\right)=-\rho D \nabla_{i} \Pi-\rho \phi(1-\phi) d^{(T)} \nabla_{i} T-d_{1} \rho \nabla_{i} \Phi_{k k}^{(1)}-d_{2} \rho \nabla_{i} \Phi_{k k}^{(2)},
\end{aligned}
$$

where the relaxation of $w_{i}$ is given by the inverse relaxation time $\xi \equiv \alpha \xi^{\prime}$ and where thermodiffusion is written in the usual way with $D^{(T)}=\phi(1-\phi) d^{(T)}$. If the relative elasticity is relaxing and the total elasticity is permanent, the relaxation parameters have to fulfill $\zeta_{l, t r}^{(11)}=\zeta_{l, t r}^{(22)}=-\zeta_{l, t r}^{(12)}$, which governs the relaxation of the former, while for the latter only vacancy diffusion is left. The relative velocity, $w_{i}$, always relaxes, because it is not related to any broken symmetry, nor to a conservation law. All other variables are conserved and show diffusional behavior.

\section{REVERSIBLE CURRENTS}

For the reversible parts of the currents in Eqs. (14) and (16)-(20) we find

$$
\begin{aligned}
Z_{i j}^{(1) R}= & \frac{1}{2} \beta_{5}^{(1)}\left(\nabla_{i} m_{j}+\nabla_{j} m_{i}\right)+\beta_{7}^{(1)}\left(U_{k j}^{(1)} \nabla_{i} m_{k}+U_{k i}^{(1)} \nabla_{j} m_{k}\right)+\beta_{6}^{(1)} m_{k} \nabla_{k} U_{i j}^{(1)} \\
& +\beta_{9}^{(1)}\left(U_{k j}^{(2)} \nabla_{i} m_{k}+U_{k i}^{(2)} \nabla_{j} m_{k}\right)+\beta_{8}^{(1)} m_{k} \nabla_{k} U_{i j}^{(2)}, \\
Z_{i j}^{(2) R}= & \frac{1}{2} \beta_{5}^{(2)}\left(\nabla_{i} m_{j}+\nabla_{j} m_{i}\right)+\beta_{7}^{(2)}\left(U_{k j}^{(2)} \nabla_{i} m_{k}+U_{k i}^{(2)} \nabla_{j} m_{k}\right)+\beta_{6}^{(2)} m_{k} \nabla_{k} U_{i j}^{(2)} \\
& +\beta_{9}^{(2)}\left(U_{k j}^{(1)} \nabla_{i} m_{k}+U_{k i}^{(1)} \nabla_{j} m_{k}\right)+\beta_{8}^{(2)} m_{k} \nabla_{k} U_{i j}^{(1)},
\end{aligned}
$$




$$
\begin{gathered}
X_{i}^{R}=\beta_{1} \nabla_{i} T+\gamma \nabla_{i}(\Pi / \rho)+\beta_{2} w_{j}\left(\nabla_{j} v_{i}+\nabla_{i} v_{j}\right)+\beta_{3} m_{j}\left(\nabla_{j} w_{i}-\nabla_{i} w_{j}\right)+\beta_{4} w_{j}\left(\nabla_{j} v_{i}-\nabla_{i} v_{j}\right) \\
-\beta_{6}^{(1)} \Phi_{k j}^{(1)} \nabla_{i} U_{k j}^{(1)}-\beta_{6}^{(2)} \Phi_{k j}^{(2)} \nabla_{i} U_{k j}^{(2)}-\beta_{8}^{(1)} \Phi_{k j}^{(1)} \nabla_{i} U_{k j}^{(2)}-\beta_{8}^{(2)} \Phi_{k j}^{(2)} \nabla_{i} U_{k j}^{(1)} \\
+\nabla_{j}\left(\beta_{5}^{(1)} \Phi_{i j}^{(1)}+\beta_{5}^{(2)} \Phi_{i j}^{(2)}+2 \beta_{7}^{(1)} \Phi_{k j}^{(1)} U_{i k}^{(1)}+2 \beta_{7}^{(2)} \Phi_{k j}^{(2)} U_{i k}^{(2)}+2 \beta_{9}^{(1)} \Phi_{k j}^{(1)} U_{i k}^{(2)}+2 \beta_{9}^{(2)} \Phi_{k j}^{(2)} U_{i k}^{(1)}\right), \\
j_{i}^{(\sigma) R}=\beta_{1} m_{i}, \\
j_{i}^{(1) R}=\gamma m_{i}, \\
\sigma_{i j}^{R}=2 \beta_{2} m_{i} w_{j} .
\end{gathered}
$$

All these reversible contributions depend on the relative velocity $w_{i}$ and vanish when there is only a single velocity. They add to the reversible $v_{i}$-dependent contributions already made apparent in Sec. IV, or they constitute new types of dynamic couplings. Nonlinear contributions are included where they contribute to the transport and convection of variables. The $\gamma$ term regulates the transport of the concentration, the $\beta_{1}$ term contributes to the transport of the entropy density, $\beta_{2}$ to the transport of the momenta, and $\beta_{4}$ is related to the convection of the relative velocity. Next, the $\beta_{5}$ contributions are related to translation, $\beta_{6}$ to transport, and $\beta_{7}$ to convection of the strain fields. The contributions $\beta_{8,9}$ couple the two strain fields dynamically.

In the most general case, the ten phenomenological reversible parameters, $\beta_{5 . .9}^{(1,2)}$, allow for ten different combinations of the two velocities in the dynamic equations or the strain fields, Eqs. (19) and (20). The most general dynamic form reads

$\dot{U}_{i j}^{(1)}+\hat{v}_{k}^{(11)} \nabla_{k} U_{i j}^{(1)}+\hat{v}_{k}^{(12)} \nabla_{k} U_{i j}^{(2)}+U_{k j}^{(1)} \nabla_{i} \breve{v}_{k}^{(11)}+U_{k i}^{(1)} \nabla_{j} \breve{v}_{k}^{(11)}+U_{k j}^{(2)} \nabla_{i} \breve{v}_{k}^{(12)}+U_{k i}^{(2)} \nabla_{j} \breve{v}_{k}^{(12)}-\frac{1}{2}\left(\nabla_{i} \tilde{v}_{j}^{(1)}+\nabla_{j} \tilde{v}_{i}^{(1)}\right)+Z_{i j}^{(1) D}=0$,

$\dot{U}_{i j}^{(2)}+\hat{v}_{k}^{(22)} \nabla_{k} U_{i j}^{(2)}+\hat{v}_{k}^{(21)} \nabla_{k} U_{i j}^{(1)}+U_{k j}^{(2)} \nabla_{i} \breve{v}_{k}^{(22)}+U_{k i}^{(2)} \nabla_{j} \breve{v}_{k}^{(22)}+U_{k j}^{(1)} \nabla_{i} \breve{v}_{k}^{(21)}+U_{k i}^{(1)} \nabla_{j} \breve{v}_{k}^{(21)}-\frac{1}{2}\left(\nabla_{i} \tilde{v}_{j}^{(2)}+\nabla_{j} \tilde{v}_{i}^{(2)}\right)+Z_{i j}^{(2) D}=0$.

The velocities $\hat{v}_{i}^{(n, m)}, \breve{v}_{i}^{(n, m)}$, and $\tilde{v}_{i}^{(n)}$, with $(n, m) \in\{1,2\}$, are linear combinations of the mean $\left(v_{i}\right)$ and the relative $\left(w_{i}\right)$ velocity, or of $v_{1}^{(1)}$ and $v_{i}^{(2)}$. Details are given in Appendix A. Cross-couplings between the two strain fields are given by the velocities $\hat{v}_{k}^{(12)}, \hat{v}_{k}^{(21)}, \breve{v}_{k}^{(12)}$, and $\breve{v}_{k}^{(21)}$, where generally we may have $\hat{v}_{k}^{(12)} \neq \hat{v}_{k}^{(21)}$ and $\breve{v}_{k}^{(12)} \neq \breve{v}_{k}^{(21)}$, while all four are proportional to the relative velocity.

Considering special cases by assigning specific values to the reversible transport parameters, Eqs. (39) and (40) for example simplify to

$$
\begin{aligned}
& \dot{U}_{i j}^{(1)}+\bar{v}_{k}^{(1)} \nabla_{k} U_{i j}^{(1)}+\bar{v}_{k}^{(3)} \nabla_{k} U_{i j}^{(2)}+U_{k j}^{(1)} \nabla_{i} \bar{v}_{k}^{(1)}+U_{k i}^{(1)} \nabla_{j} \bar{v}_{k}^{(1)}+U_{k j}^{(2)} \nabla_{i} \bar{v}_{k}^{(3)}+U_{k i}^{(2)} \nabla_{j} \bar{v}_{k}^{(3)}-\frac{1}{2}\left(\nabla_{i} \bar{v}_{j}^{(1)}+\nabla_{j} \bar{v}_{i}^{(1)}\right)+Z_{i j}^{(1) D}=0, \\
& \dot{U}_{i j}^{(2)}+\bar{v}_{k}^{(2)} \nabla_{k} U_{i j}^{(2)}+\bar{v}_{k}^{(3)} \nabla_{k} U_{i j}^{(1)}+U_{k j}^{(2)} \nabla_{i} \bar{v}_{k}^{(2)}+U_{k i}^{(2)} \nabla_{j} \bar{v}_{k}^{(2)}+U_{k j}^{(1)} \nabla_{i} \bar{v}_{k}^{(3)}+U_{k i}^{(1)} \nabla_{j} \bar{v}_{k}^{(3)}-\frac{1}{2}\left(\nabla_{i} \bar{v}_{j}^{(2)}+\nabla_{j} \bar{v}_{i}^{(2)}\right)+Z_{i j}^{(2) D}=0,
\end{aligned}
$$

with three linear combinations $\bar{v}_{k}^{(1,2,3)}$ of $v_{i}$ and $w_{i}$. The first two, $\bar{v}_{k}^{(1)}$ and $\bar{v}_{k}^{(2)}$, enter the translation, transport, and convection of $U_{i j}^{(1)}$ and $U_{i j}^{(2)}$, respectively, while $\bar{v}_{k}^{(3)}$ occurs in the coupling terms between them (see again Appendix A). Even simpler is the "naive" model, where translation, transport, and convection occur through the velocities, $v_{i}^{(1)}$ and $v_{i}^{(2)}$, associated with the appropriate translation fields,

$$
\dot{U}_{i j}^{(n)}+v_{k}^{(n)} \nabla_{k} U_{i j}^{(n)}+U_{k j}^{(n)} \nabla_{i} v_{k}^{(n)}+U_{k i}^{(n)} \nabla_{j} v_{k}^{(n)}-\frac{1}{2}\left(\nabla_{i} v_{j}^{(n)}+\nabla_{j} v_{i}^{(n)}\right)+Z_{i j}^{(n) D}=0
$$

with either $n=1$ or $n=2$. There is no dynamic coupling between systems 1 and 2. All $\beta$ parameters are fixed (cf. Appendix A).

With only one velocity $v_{i}$ (the relative velocity $w_{i}$ being relaxed to zero) only the trivial model

$$
\dot{U}_{i j}^{(n)}+v_{k} \nabla_{k} U_{i j}^{(n)}+U_{k j}^{(n)} \nabla_{i} v_{k}+U_{k i}^{(n)} \nabla_{j} v_{k}-A_{i j}+Z_{i j}^{(n) D}=0
$$

is possible. This has to be compared with Eqs. (52) and (53) of Ref. [8], rendering the parameters there to be $a=1$ and $b^{x}=1$.

Another set of choices for the $\beta$ parameters leads to a four-parameter model with no reversible dynamic coupling between the total and the relative strains

$$
\begin{gathered}
\dot{U}_{i j}^{(\mathrm{tot})}+\bar{v}_{k}^{(4)} \nabla_{k} U_{i j}^{(\mathrm{tot})}+U_{k j}^{(\mathrm{tot})} \nabla_{i} \bar{v}_{k}^{(5)}+U_{k i}^{(\mathrm{tot})} \nabla_{j} \bar{v}_{k}^{(5)}-\frac{1}{2}\left(\nabla_{i} \bar{v}_{j}^{(6)}+\nabla_{j} \bar{v}_{i}^{(6)}\right)+Z_{i j}^{(\mathrm{tot}) \mathrm{D}}=0, \\
\dot{U}_{i j}^{(\mathrm{rel})}+\bar{v}_{k}^{(4)} \nabla_{k} U_{i j}^{(\mathrm{rel})}+U_{k j}^{(\mathrm{rel})} \nabla_{i} \bar{v}_{k}^{(5)}+U_{k i}^{(\mathrm{rel})} \nabla_{j} \bar{v}_{k}^{(5)}-\frac{1}{2}\left(\nabla_{i} \bar{v}_{j}^{(7)}+\nabla_{j} \bar{v}_{i}^{(7)}\right)+Z_{i j}^{(\mathrm{rel}) D}=0
\end{gathered}
$$

(see once more, Appendix A). 


\section{RELATIVE ROTATIONS}

While elastic strains are described by symmetric two-rank tensors, rotations are related to antisymmetric second-rank tensors, $\Omega_{i j} \equiv-\Omega_{j i}$. In linear order, rotations of the two elastic media are related to the two translation vectors by $2 \Omega_{i j}^{(n)}=$ $\nabla_{i} u_{j}^{(n)}-\nabla_{j} u_{i}^{(n)}$. Only relative rotations $2 \Omega_{i j}^{(\mathrm{rel})}=\nabla_{i} u_{j}^{(\mathrm{rel})}-$ $\nabla_{j} u_{i}^{\text {(rel) }}$ can be macroscopic variables. Relative rotations are a common concept in anisotropic elastomers, e.g., nematic ones, where rotations of the preferred direction relative to the elastic network are important. For a nonlinear description of relative rotations, compare Refs. [29-31].

The Gibbs relation, Eq. (1), has to be amended by

$$
d \varepsilon=\ldots+W_{i j}^{(\mathrm{rel})} d \Omega_{i j}^{(\mathrm{rel})}
$$

thereby defining the conjugate field $W_{i j}^{(\text {rel })}$. There is an additional $-W_{i j}^{(\mathrm{rel})} d \Omega_{i j}^{(\mathrm{rel})}$ to the pressure differential in Eq. (3) and another symmetry relation $\Omega_{i k}^{(\mathrm{rel})} W_{k j}^{(\mathrm{rel})}=\Omega_{j k}^{(\mathrm{rel})} W_{k i}^{(\mathrm{rel})}$.

In the statics of the considered isotropic materials, the conjugate of relative rotations, $W_{i j}^{(\mathrm{rel})}$, is given by

$$
W_{i j}^{(\mathrm{rel})}=D_{\Omega} \Omega_{i j}^{(\mathrm{rel})} \text {. }
$$

This equation shows that there is one static response coefficient $\left(D_{\Omega}\right)$ that quantifies the overall energetic contribution of relative rotations by themselves, relating relative rotations, $\Omega_{i j}^{(\mathrm{rel})}$, to the associated thermodynamic force, $W_{i j}^{(\mathrm{rel})}$. It describes the stiffness of relative rotations and can be seen, for example, as the analog of the static relation between elastic stresses and strain fields, Eqs. (10) and (11).

The dynamic equation is written in terms of the mean velocity

$$
\begin{aligned}
& \dot{\Omega}_{i j}^{(\mathrm{rel})}+v_{k} \nabla_{k} \Omega_{i j}^{(\mathrm{rel})}+\Omega_{k j}^{(\mathrm{rel})} \nabla_{i} v_{k} \\
& \quad-\Omega_{k i}^{(\mathrm{rel})} \nabla_{j} v_{k}+Q_{i j}^{(\mathrm{rel}) R}+Q_{i j}^{(\mathrm{rel}) D}=0,
\end{aligned}
$$

which facilitates the fulfillment of the thermodynamic condition

$$
R^{\Omega}=\ldots+W_{i j}^{(\mathrm{rel})} Q_{i j}^{(\mathrm{rel}) *} \geqslant 0
$$

with $* \in\{R, D\}$, and where the $\ldots$ stand for the contributions given in Eq. (24). In particular, the (reversible) transport term is compensated by all other transport contributions, and the convective term requires an additional contribution to the stress tensor, Eq. $(21), \sigma_{i j}^{(\text {conv })}=\ldots+W_{j k}^{(\text {rel })} \Omega_{i k}^{(\text {rel })}+$ $W_{i k}^{(\mathrm{rel})} \Omega_{j k}^{(\mathrm{rel})}$.

The dissipative current only contains the relaxation of the relative rotations without any cross-coupling,

$$
Q_{i j}^{(\mathrm{rel}) D}=\tau_{\Omega} W_{i j}^{(\mathrm{rel})}
$$

For the reversible current we find

$$
\begin{aligned}
Q_{i j}^{(\mathrm{rel}) R}= & \beta_{4}^{(Q)}\left(\nabla_{i} m_{j}-\nabla_{j} m_{i}\right)+\beta_{5}^{(Q)}\left(m_{i} \nabla_{j} T-m_{j} \nabla_{i} T\right)+\beta_{6}^{(Q)}\left(m_{i} \nabla_{j} \Pi-m_{j} \nabla_{i} \Pi\right) \\
& +\beta_{7}^{(Q)}\left(m_{i} \nabla_{k} \Phi_{k j}^{(1)}-m_{j} \nabla_{k} \Phi_{k i}^{(1)}\right)+\beta_{8}^{(Q)}\left(m_{i} \nabla_{k} \Phi_{k j}^{(2)}-m_{j} \nabla_{k} \Phi_{k i}^{(2)}\right) \\
& +\beta_{9}^{(Q)} m_{k}\left(\nabla_{i} \Phi_{k j}^{(1)}-\nabla_{j} \Phi_{k i}^{(1)}\right)+\beta_{10}^{(Q)} m_{k}\left(\nabla_{i} \Phi_{k j}^{(2)}-\nabla_{j} \Phi_{k i}^{(2)}\right)+\beta_{14}^{(Q)} m_{k} \nabla_{k} \Omega_{i j}^{(\mathrm{rel})} \\
& +\beta_{15}^{(Q)}\left(\Omega_{i k}^{(\mathrm{rel})} \nabla_{j} m_{k}-\Omega_{j k}^{(\mathrm{rel})} \nabla_{i} m_{k}\right)+\beta_{16}^{(Q)}\left(U_{k j}^{(1)} \nabla_{i} m_{k}-U_{k i}^{(1)} \nabla_{j} m_{k}\right)+\beta_{17}^{(Q)}\left(U_{k j}^{(2)} \nabla_{i} m_{k}-U_{k i}^{(2)} \nabla_{j} m_{k}\right)
\end{aligned}
$$

Due to the thermodynamic condition Eq. (50), the reversible currents give rise to the following additional terms in the other reversible currents:

$$
\begin{gathered}
j_{i}^{(\sigma) R}=\ldots+2 \beta_{5}^{(Q)} m_{j} W_{j i}^{(\mathrm{rel})}, \\
j_{i}^{(1) R}=\ldots+2 \beta_{6}^{(Q)} m_{j} W_{j i}^{(\mathrm{rel})}, \\
X_{i}^{R}=\ldots+2 \beta_{4}^{(\Omega)} \nabla_{j} W_{j i}^{(\mathrm{rel})}-\beta_{14}^{(Q)} W_{k j}^{(\mathrm{rel})} \nabla_{i} \Omega_{k j}^{(\mathrm{rel})}+2 \beta_{15}^{(Q)} \nabla_{k}\left(\Omega_{i j}^{(\mathrm{rel})} W_{k j}^{(\mathrm{rel})}\right) \\
+2 \beta_{16}^{(Q)} \nabla_{k}\left(U_{i j} W_{k j}^{(\mathrm{rel})}\right)+2 \beta_{17}^{(Q)} \nabla_{k}\left(U_{i j}^{(\mathrm{rel})} W_{k j}^{(\mathrm{rel})}\right), \\
Z_{i j}^{(1) R}=\ldots+\beta_{7}^{(Q)}\left(\nabla_{j} m_{k} W_{k i}^{(\mathrm{rel})}+\nabla_{i} m_{k} W_{k j}^{(\mathrm{rel})}\right)+\beta_{9}^{(Q)} \nabla_{k}\left(m_{i} W_{k j}^{(\mathrm{rel})}+m_{j} W_{k i}^{(\mathrm{rel})}\right), \\
Z_{i j}^{(2) R}=\ldots+\beta_{8}^{(Q)}\left(\nabla_{j} m_{k} W_{k i}^{(\mathrm{rel})}+\nabla_{i} m_{k} W_{k j}^{(\mathrm{rel})}\right)+\beta_{10}^{(Q)} \nabla_{k}\left(m_{i} W_{k j}^{(\mathrm{rel})}+m_{j} W_{k i}^{(\mathrm{rel})}\right),
\end{gathered}
$$

which supplement the expressions given in Eqs. (33)-(37).

Through the reversible contributions in Eq. (52), the translation of $Q_{i j}^{(\mathrm{rev})}$ occurs with the velocity $\bar{v}_{i}^{(\mathrm{lq})} \equiv \beta_{4}^{(Q)} \alpha w_{i}$, the effective transport velocity is $\bar{v}_{i}^{(\mathrm{tq})} \equiv v_{i}+\beta_{14}^{(Q)} \alpha w_{i}$, and the convective velocity is $\bar{v}_{i}^{(\mathrm{cq})} \equiv v_{i}+\beta_{15}^{(Q)} \alpha w_{i}$ with $\alpha=\phi(1-\phi) \rho$. Thus, the dynamic equation for $\Omega_{i j}^{(\mathrm{rel})}$ can be written as

$$
\dot{\Omega}_{i j}^{(\mathrm{rel})}-\left(\nabla_{j} \bar{v}_{i}^{(\mathrm{lq})}-\nabla_{i} \bar{v}_{j}^{(\mathrm{lq})}\right)+\bar{v}_{k}^{(\mathrm{tq})} \nabla_{k} \Omega_{i j}^{(\mathrm{rel})}+\Omega_{k j}^{(\mathrm{rel})} \nabla_{i} \bar{v}_{k}^{(\mathrm{cq})}-\Omega_{k i}^{(\mathrm{rel})} \nabla_{j} \bar{v}_{k}^{(\mathrm{cq})}+\tilde{Q}_{i j}^{(\mathrm{rel}) R}+Q_{i j}^{(\mathrm{rel}) D}=0
$$

with $\tilde{Q}_{i j}^{(\text {rel }) R}$ given by $Q_{i j}^{(\text {rel }) R}$, Eq. (52), without the $\beta_{4}^{(Q)}, \beta_{14}^{(Q)}$, and $\beta_{15}^{(Q)}$ terms. If there is only one velocity (the relative velocity being relaxed to zero), comparison with Eq. (55) of Ref. [8] reveals that the parameter $a$ there has to be $a=1$. 


\section{RELATIVE TRANSLATIONS}

A homogeneous translation of a single-elastic medium does not change the internal state of the system and is therefore not a macroscopic variable. Homogeneous translations of the individual systems in a two-component elastic medium, $u_{i}^{(1)}$ and $u_{i}^{(2)}$, do change the internal state, if $u_{i}^{(1)} \neq u_{i}^{(2)}$. Thus, relative translations $u_{i}^{(\mathrm{rel})} \equiv u_{i}^{(1)}-u_{i}^{(2)}$ are to be put on the list of variables. These variables are neither related to a conservation law nor to a broken symmetry, and therefore relax in a finite time. They are relevant, if the relaxation times are in the time range of the other (hydrodynamic) variables. In addition, these variables are not covered by relative strains, $U_{i j}^{(\mathrm{rel})}$, because the latter cannot describe homogeneous translations.

We amend the Gibbs relation in Eq. (1), defining the conjugate force $\Phi_{i}^{(\text {rel })}$, via

$$
d \varepsilon=\ldots+\Phi_{i}^{(\mathrm{rel})} d u_{i}^{(\mathrm{rel})}
$$

where the ... represent all other variables. This also leads to an additional term in the Gibbs-Duhem relation, Eq. (3), $d p=\ldots-\Phi_{i}^{(\mathrm{rel})} d u_{i}^{(\mathrm{rel})}$, and to an additional symmetry relation $u_{i}^{(\mathrm{rel})} \Phi_{j}^{(\mathrm{rel})}=u_{j}^{(\mathrm{rel})} \Phi_{i}^{(\mathrm{rel})}$ to guarantee rotational invariance of the energy functional.

The statics of these degrees of freedom are simply given by

$$
\Phi_{i}^{(\mathrm{rel})}=c_{d} u_{i}^{(\mathrm{rel})}
$$

with a single coefficient $c_{d}$.

In simplest approximation, the time evolution for homogeneous translations is $\dot{u}_{i}^{(1,2)}=v_{i}^{(1,2)}$, leading to the dynamic equation for relative translations

$$
\dot{u}_{i}^{(\mathrm{rel})}+z_{i}^{(\mathrm{rel}) R}+z_{i}^{(\mathrm{rel}) D}=0
$$

where $z_{i}^{\text {(rel) }}$ contains the phenomenological couplings to other variables. Thermodynamics requires

$$
R=\ldots+\Phi_{i}^{(\mathrm{rel})} z_{i}^{(\mathrm{rel})} \geqslant 0
$$

for dissipative ( $>$ ) and reversible (=) contributions; the ... are the parts given in Eqs. (24) and (50).

Using the standard symmetry requirements and the assumption that $\Phi_{i}^{(\mathrm{rel})}$ is homogeneous (as is $u_{i}$ ), we get

$$
\begin{gathered}
z_{i}^{(\mathrm{rel}) R}=-\beta_{1}^{(\mathrm{rt})} m_{i}, \\
X_{i}^{R}=\ldots+\beta_{1}^{(\mathrm{rt})} \Phi_{i}^{(\mathrm{rel})},
\end{gathered}
$$

where $X_{i}^{R}$ is the reversible current of the relative velocity and the $\ldots$ are given by Eqs. (35) and (55). We will choose $\beta_{1}^{(\mathrm{rt})}=$ $1 / \alpha$, in order to comply with the naive model $\dot{u}_{i}^{(1,2)}=v_{i}^{(1,2)}$.

For the dissipative part we find cross-couplings to the time evolution of the thermal degree of freedom and to the concentration dynamics

$$
\begin{gathered}
z_{i}^{(\mathrm{rel}) D}=\zeta^{(\mathrm{rt})} \Phi_{i}^{(\mathrm{rel})}-\kappa_{2}^{(\mathrm{rt})} \nabla_{i} T-d_{2}^{(\mathrm{rt})} \nabla_{i} \Pi, \\
j_{i}^{(\sigma) D}=\ldots-\kappa_{2}^{(\mathrm{rt})} \Phi_{i}^{(\mathrm{rel})} \\
j_{i}^{(1) D}=\ldots-d_{2}^{(\mathrm{rt})} \Phi_{i}^{(\mathrm{rel})}
\end{gathered}
$$

where the ... are given in Eqs. (27) and (32), respectively.
Note that the dynamic equation for $u_{i}^{(\text {rel })}$ is fully linear, e.g., a transport term $\sim \nabla_{k} u_{i}^{(\mathrm{rel})}$ is absent, since we only consider homogeneous relative translations. This is not a serious restriction, because for physical reasons such relative translations have to be small. Moreover, inhomogeneous translations $\nabla_{j} u_{i}$ are already taken into account by strain variables and by the relative rotations. The linear nature of the $u_{i}^{(\mathrm{rel})}$ dynamics also avoids any problems connected with the possible use of the displacement vector in order to describe nonlinear strains or nonlinear relative rotations.

\section{ILLUSTRATIVE MINIMAL EXAMPLES}

The central step of the present work is to extend a previous macroscopic dynamic characterization of elastic or viscoelastic composite systems [8] by including separate velocity fields for each component. As it turns out, this extension is necessary already to describe very basic intuitive dynamic processes, as the two following minimal example situations are intended to illustrate.

For this purpose, we shall reduce the above equations to a minimum by confining ourselves to corresponding basic scenarios. Accordingly, as a first and very common approximation, we address so-called incompressible systems. This implies $\kappa_{\mu} \rightarrow 0$ for the overall composite system in Eq. (4). Tiny changes in density $\delta \rho$ then imply huge changes in pressure via the chemical potential $\mu$ in Eq. (7) and according to Eq. (1). Through Eqs. (15) and (17), the density $\rho$ is then regulated to remain approximately constant. For $\kappa_{\mu} \rightarrow 0$, this process occurs sufficiently quickly so that we can consider it as happening on a different scale, setting $\delta \rho=0$ in our evaluations. Consequently, Eq. (15) drops out and only requires the common relation $\nabla_{i} v_{i}=0$.

Moreover, couplings to variations in entropy density $\delta \sigma$ and temperature $T$ as well as the dynamics of entropy density variations are not taken into account. Formally, this means that $\alpha_{\phi} \rightarrow \infty, \alpha_{\rho} \rightarrow \infty, \alpha_{1} \rightarrow \infty$, and $\alpha_{2} \rightarrow \infty$ in Eqs. (4)-(9). Similarly, in the dissipation function and dissipative currents in Eqs. (25)-(32), we set $\kappa_{1}=\kappa_{2}=0$ and $d^{(T)}=0$. The same applies to $\kappa_{2}^{(\mathrm{rt})}=0$ in Eqs. (65) and (66). Likewise, the reversible couplings to temperature gradients are not taken into account, setting $\beta_{1}=0$ in Eqs. (35) and (36). Consequently, the dynamic equation for variations in entropy density, Eq. (14), is not evaluated. Furthermore, we do not involve the effects of relative rotations summarized in Sec. VII.

Instead, we focus on the remaining key dynamic quantities. Here, these are the mean velocity $v_{i}$ and the relative velocity $w_{i}$ (which is equivalent to considering the velocities $v_{i}^{(1)}$ and $v_{i}^{(2)}$ of the two components as explained above), the two strains $U_{i j}^{(1)}$ and $U_{i j}^{(2)}$ of the two-component elastic composite material, relative displacements $u_{i}^{(\mathrm{rel})}$ between the two components, and possibly the concentration $\phi$. It appears reasonable to first consider a fundamental but very important spatially homogeneous dynamic situation. Afterwards, in our second minimal example, we address a basic minimal dynamic scenario that involves spatial variations. 


\section{A. Swinging in a simple elastic composite material}

In our first example, we step back to very basic elastic composite materials consisting, e.g., of spherical inclusions as a second component in an elastic matrix as a first component. Both components should be perfectly permanently elastic, that is, their deformations are completely reversible. Thus, we impose $\zeta_{t r}^{(m n)}=0$ and $\zeta_{l}^{(m n)}=0[(m n) \in\{(11),(12),(22)\}]$ in Eqs. (28) and (29). The same applies to their mutual purely translational coupling, which should be permanently elastic as well, so that we have $\zeta^{(\mathrm{rt})}=0$ in Eq. (65) for the relative translations. In the following, we may even consider the second component to be perfectly rigid, which can be taken into account by setting $c_{l}^{(22)} \rightarrow \infty$ and $c_{t r}^{(22)} \rightarrow \infty$ in Eqs. (4)-(11).

Searching for spatially homogeneous solutions, the system of dynamic equations in Secs. IV and VIII for the relevant remaining variables becomes

$$
\begin{gathered}
\dot{\phi}=0, \\
\dot{g}_{i}=0, \\
\dot{w}_{i}+\xi^{\prime} \alpha w_{i}+\beta_{1}^{(\mathrm{rt})} c_{d} u_{i}^{(\mathrm{rel})}=0, \\
\dot{U}_{i j}^{(1)}=0, \\
\dot{U}_{i j}^{(2)}=0, \\
\dot{u}_{i}^{(\mathrm{rel})}-\beta_{1}^{(\mathrm{rt})} \alpha w_{i}=0,
\end{gathered}
$$

where here $\alpha=\phi(1-\phi) \rho$ remains constant. Only two of these dynamic equations are nontrivial, and we may combine them. Solving the last equation for $w_{i}$ and inserting it into Eq. (70), we find a decoupled dynamic equation for $u_{i}^{\text {(rel) }}$,

$$
\ddot{u}_{i}^{(\mathrm{rel})}+\xi^{\prime} \alpha \dot{u}_{i}^{(\mathrm{rel})}+\left(\beta_{1}^{(\mathrm{rt})}\right)^{2} c_{d} \alpha u_{i}^{(\mathrm{rel})}=0 .
$$

Obviously, this represents the dynamic equation for a damped harmonic oscillator. It describes the mutual swinging of the two components against each other. Depending on the actual values of the material parameters, all the different scenarios for a harmonic oscillator can be observed. Illustratively, the damping here naturally evolves from the friction arising when the two subsystems move relatively to, or "through" each other, described by the inverse relaxation time $\xi=\xi^{\prime} \alpha$ in Eq. (31).

For the oscillations to become effective, initially a relative translation or relative velocity needs to be excited. One could, for example, mechanically shake from outside the elastic matrix, that is, our first component. If the inclusions are of significantly higher density, their buoyant effect retards their motion, initiating a relative translation. Another possibility would be to apply for magnetic inclusions an impulse of an external magnetic field gradient to drag them against the surrounding elastic matrix.

We add a remark on an interesting peculiarity arising in this context of rigid inclusions swinging against a surrounding elastic environment. It further stresses the importance and necessity of the variables of relative translations. When rigid inclusions swing relatively to the center of mass of a surrounding elastic matrix, they naturally distort the matrix.
Nevertheless, we here find that the macroscopic strain $U_{i j}^{(1)}$ of the elastic matrix remains constant [see Eq. (71)]. This can be understood from the following mesoscopic point of view. Moving a rigid inclusion under no-slip surface conditions against a surrounding elastic medium, it contracts the medium along its axis of motion at the front and extends it at the rear $[45,46]$. The magnitudes of deformation are symmetric with respect to the front and the rear. Therefore, on the macroscopic level described by $U_{i j}^{(1)}$, the mesoscopic strains of opposite sign at the front and at the rear of the sphere average out. Instead, the relative translations naturally incorporate this mode of relative displacement into the macroscopic characterization.

\section{B. Actuation or active stress by one component}

To proceed one step beyond the simple picture above, we now include basic spatial variations. Moreover, we consider one of the two components to exert an actuating or active stress onto the system. For example, we may think of a magnetic second component that contracts or elongates along the direction of an externally imposed magnetic field, particularly in the context of soft magnetic actuators. We may also consider an active component that induces mechanical stresses by consuming chemicals. Examples are filamentous networks in biological cells that actively contract in the cell environment, or muscle cells surrounded by other biological tissue.

The components of the associated actuating or active stress $\Phi_{i j}^{\text {act (2) }}$ are added to $\Phi_{k k}^{(2)}$ and $\tilde{\Phi}_{i j}^{(2)}$ in Eqs. (9) and (11), respectively. This implies that they can be added via a contribution $\Phi_{i j}^{\text {act (2) }} d U_{i j}^{(2)}$ to the Gibbs relation in Eq. (1). Similarly, a contribution $\Phi_{i j}^{\text {act (2) }} U_{i j}^{(2)}$ to the total energy density in Eq. (4) results.

The stress $\Phi_{i j}^{\text {act (2) }}$ will initiate relative and possibly overall distortions of the whole system, unless the system is overly confined. It is important to note that in reality such distortions will frequently start from spatial heterogeneities of the system, particularly from its boundaries [47], and then propagate into the bulk. This requires one to consider more specific systems and situations. Instead, our scope here is to illustrate on a basic minimal example the general interplay especially between the newly coupled macroscopic variables of strain, $U_{i j}^{(1)}, U_{i j}^{(2)}$, and relative velocity, $w_{i}$. Therefore, we rather concentrate on the bulk and impose a spatially modulated actuation along one direction, here the $x$ direction, so that

$$
\Phi_{i j}^{\mathrm{act}(2)}=P^{\mathrm{act}}(t) \sin (k x) \delta_{i x} \delta_{j x},
$$

where $P^{\text {act }}(t)$ contains the magnitude of active or actuation stress, while $k$ denotes the wave number of spatial modulation. In experiments, such modulations could, for instance, be achieved through imposed patterns of light irradiation in light-activated systems relative to the daylight reference state $[48,49]$ or through spatially controlled release of nutrients in biological systems.

Again, we consider the overall mass density to be conserved, $\dot{\rho}=0$, so that $\nabla_{i} v_{i}=0$, and we neglect couplings to variations in entropy density $\delta \sigma$. Assuming that $\Phi_{x x}^{\text {act (2) }}$ only induces small deviations from the initial state, we radically linearize the remaining dynamic Eqs. (16)-(20) and (61). In 
addition, we assume that the system still can be treated as isotropic. Again not taking into account relative rotations, only including the leading contribution $\xi^{\prime} m_{i}=\xi w_{i}$ in the dissipative current $X_{i}^{D}$ in Eq. (31), that is, neglecting the contributions $\sim v_{i j k l}^{(c)}$ and $\sim v_{i j k l}^{(w)}$, the dynamic equations then read

$$
\begin{gathered}
\dot{\phi}=-\frac{\alpha}{\rho}(1+\gamma) \nabla_{i} w_{i}-\frac{1}{\rho} \nabla_{i} j_{i}^{(1) D} \\
\dot{v}_{i}=-\frac{1}{\rho} \nabla_{i} p+\frac{1}{2 \rho} \nabla_{j}\left(\Phi_{i j}^{(1)}+\Phi_{i j}^{(2)}\right)-\frac{1}{\rho} \nabla_{j} \sigma_{i j}^{D}, \\
\dot{w}_{i}=-\frac{1}{\rho}(1+\gamma) \nabla_{i} \Pi-\beta_{5}^{(1)} \nabla_{j} \Phi_{i j}^{(1)} \\
-\beta_{5}^{(2)} \nabla_{j} \Phi_{i j}^{(2)}-c_{d} \beta_{1}^{(\mathrm{rt})} u_{i}^{(\mathrm{rel})}-X_{i}^{D}, \\
\dot{U}_{i j}^{(1)}=A_{i j}-\frac{1}{2} \alpha \beta_{5}^{(1)}\left(\nabla_{i} w_{j}+\nabla_{j} w_{i}\right)-Z_{i j}^{(1) D}, \\
\dot{U}_{i j}^{(2)}=A_{i j}-\frac{1}{2} \alpha \beta_{5}^{(2)}\left(\nabla_{i} w_{j}+\nabla_{j} w_{i}\right)-Z_{i j}^{(2) D}, \\
\dot{u}_{i}^{(\mathrm{rel})}=-\alpha \beta_{1}^{(\mathrm{rt})} w_{i}-z_{i}^{(\mathrm{rel}) D},
\end{gathered}
$$

where

$$
\begin{gathered}
\Pi=\frac{1}{\kappa_{\phi}} \delta \phi+\frac{1}{\rho \kappa_{u 1}} U_{k k}^{(1)}+\frac{1}{\rho \kappa_{u 2}} U_{k k}^{(2)} \\
\Phi_{i j}^{(1)}=c_{t r}^{(11)} U_{i j}^{(1)}+c_{t r}^{(12)} U_{i j}^{(2)} \\
+\frac{1}{3} \delta_{i j}\left(\bar{c}^{(11)} U_{k k}^{(1)}+\bar{c}^{(12)} U_{k k}^{(2)}+\frac{1}{\rho \kappa_{u 1}} \delta \phi\right), \\
\Phi_{i j}^{(2)}=c_{t r}^{(22)} U_{i j}^{(2)}+c_{t r}^{(12)} U_{i j}^{(1)} \\
+\frac{1}{3} \delta_{i j}\left(\bar{c}^{(22)} U_{k k}^{(2)}+\bar{c}^{(12)} U_{k k}^{(1)}+\frac{1}{\rho \kappa_{u 2}} \delta \phi\right)+\Phi_{i j}^{\mathrm{act}(2)},
\end{gathered}
$$

while $\bar{c}^{(m n)}=c_{l}^{(m n)}-c_{t r}^{(m n)}$ for $(m n) \in\{(11),(12),(22)\}$. The remaining parts of the dissipative currents read $j_{i}^{(1) D}=-\rho d \nabla_{i} \Pi-d_{1} \nabla_{i} \Phi_{k k}^{(1)}-d_{2} \nabla_{i} \Phi_{k k}^{(2)}-d_{2}^{(\mathrm{rt})} c_{d} u_{i}^{(\mathrm{rel})}, \sigma_{i j}^{D}$ $=-v_{i j k l} \nabla_{l} v_{k}, X_{i}^{D}=\alpha \xi^{\prime} w_{i}$ while $Z_{i j}^{(1) D}$ and $Z_{i j}^{(2) D}$ are given by Eqs. (28) and (29), respectively, where we neglect stress diffusion and again couplings to temperature setting $\xi_{t r}^{(m n)}=$ $\xi_{l}^{(m n)}=0$ for $(m n) \in\{(11),(12),(22)\}$ and $\kappa_{1}=\kappa_{2}=0$, as well as $z_{i}^{(\mathrm{rel}) D}=c_{d} \zeta^{(\mathrm{rt})} u_{i}^{(\mathrm{rel})}-d_{2}^{(\mathrm{rt})} \nabla_{i} \Pi$.

To be able to consider an analytically traceable situation, we now confine all spatial variations and thus the distortions and dynamics to the $x$ direction. Together with the abovementioned overall volume conservation, $\nabla_{i} v_{i}=0$, we obtain $v_{i}=0$ and thus $A_{i j}=0$.

Interesting aspects arise from the explicit consideration of the relative velocity $w_{i}$ between the two components. Focusing only on the reversible parts, that is, not considering dissipative effects, we first rewrite the above equations for $v_{i}=0$ as

$$
\begin{gathered}
\dot{\phi}=-\frac{\alpha}{\rho}(1+\gamma) \partial_{x} w_{x} \\
\dot{w}_{x}=-\frac{1}{\rho}(1+\gamma) \partial_{x} \Pi-\beta_{5}^{(1)} \partial_{x} \Phi_{x x}^{(1)} \\
-\beta_{5}^{(2)} \partial_{x} \Phi_{x x}^{(2)}-c_{d} \beta_{1}^{(\mathrm{rt})} u_{x}^{(\mathrm{rel})} \\
\dot{U}_{x x}^{(1)}=-\alpha \beta_{5}^{(1)} \partial_{x} w_{x} \\
\dot{U}_{x x}^{(2)}=-\alpha \beta_{5}^{(2)} \partial_{x} w_{x} \\
\dot{u}_{x}^{(\mathrm{rel})}=-\alpha \beta_{1}^{(\mathrm{rt})} w_{x} .
\end{gathered}
$$

From here, we see that changes in concentration $\phi$, in the strains $U_{x x}^{(1)}$ and $U_{x x}^{(2)}$ of the two components, and in their mutual relative displacement $u_{x}^{(\mathrm{rel})}$ are in this case solely due to reversible transport through the relative velocity $w_{x}$ between the two components. That is, the actuation or active stress drives the relative velocity, not directly the strains. The strains and the other variables are only indirectly driven by the actuation or active stress through the relative velocity $w_{i}$.

This set of equations can be solved by taking another time derivative of Eq. (86) to then insert Eqs. (85) and (87)-(89). For example, for an oscillating actuation or activation stress

$$
\Phi_{x x}^{\text {act }(2)}=P^{\text {act }} \sin (k x) \cos (\omega t)
$$

of frequency $\omega$, we obtain

$$
\begin{gathered}
\delta \phi=-\frac{\alpha}{\rho}(1+\gamma) \frac{k}{\omega} P_{w} \sin (k x) \cos (\omega t), \\
w_{x}=P_{w} \cos (k x) \sin (\omega t), \\
U_{x x}^{(1)}=-\alpha \beta_{5}^{(1)} \frac{k}{\omega} P_{w} \sin (k x) \cos (\omega t), \\
U_{x x}^{(2)}=-\alpha \beta_{5}^{(2)} \frac{k}{\omega} P_{w} \sin (k x) \cos (\omega t), \\
u_{x}^{(\mathrm{rel})}=\alpha \beta_{1}^{(\mathrm{rt})} \frac{1}{\omega} P_{w} \cos (k x) \cos (\omega t),
\end{gathered}
$$

while

$$
\begin{aligned}
P_{w}= & -\beta_{5}^{(2)} k \omega P^{\mathrm{act}}\left\{c_{d} \alpha\left(\beta_{1}^{(\mathrm{rt})}\right)^{2}+\omega^{2}-k^{2}\right. \\
& \times\left[\frac{(1+\gamma)^{2} \alpha}{\rho^{2} \kappa_{\phi}}+\frac{\alpha \beta_{5}^{(1)}}{\rho^{2} \kappa_{u 1}}+\frac{\alpha \beta_{5}^{(2)}}{\rho^{2} \kappa_{u 2}}\right. \\
& +\alpha\left(\beta_{5}^{(1)}\right)^{2} \tilde{c}^{(11)}+\alpha\left(\beta_{5}^{(2)}\right)^{2} \tilde{c}^{(22)}+2 \alpha \beta_{5}^{(1)} \beta_{5}^{(2)} \tilde{c}^{(12)} \\
& \left.\left.+\frac{\alpha \beta_{5}^{(1)}(1+\gamma)}{3 \rho^{2} \kappa_{u 1}}+\frac{\alpha \beta_{5}^{(2)}(1+\gamma)}{3 \rho^{2} \kappa_{u 2}}\right]\right\}^{-1},
\end{aligned}
$$

with $\tilde{c}^{(m n)}=(2 / 3) c_{t r}^{(m n)}+(1 / 3) c_{l}^{(m n)}$ for $(m n) \in\{(11),(12)$, (22)\}. Taking the limit $\omega \rightarrow 0$ in Eqs. (90)-(96), we obtain the limits of static concentration changes $\delta \phi$, static distortions $U_{x x}^{(1)}$ and $U_{x x}^{(2)}$, as well as static relative displacements $u_{x}^{(\text {rel })}$ in response to the actuation or active stresses $\Phi_{x x}^{\text {act (2) }}=$ $P^{\text {act }} \sin (k x)$. Obviously, if stretching the second component implies contraction of the first component along the $x$ direction due to overall volume conservation, then $\beta_{5}^{(1)}$ and $\beta_{5}^{(2)}$ should be of opposite sign. 
Including in Eq. (86) the dissipative current $X_{i}^{D}=\alpha \xi^{\prime} w_{i}$ [see Eq. (78)] involves an additional damping of the relative velocity. With this extension, taking again the time derivative of Eq. (86) before inserting Eqs. (85) and (87)-(89), we obtain at each position $x$ an equation for $w_{x}$ that resembles the one for a damped driven harmonic oscillator. Corresponding considerations apply (see also our conclusions above in Sec. IX A). This cures the issue of the possible resonance frequency that may arise from Eq. (96). We also note that the incorporation of dissipative effects leads to phase shifts when compared to the thermodynamic driving force and to imaginary parts of the response functions.

The further dissipative currents include contributions that describe deviations from a perfectly elastic and thus reversibly deformable system. For instance, $j_{i}^{(1) D}$ in Eq. (76) contains diffusional relocations of one of the two components relative to the other. This is in contrast to the reversible transport through the relative velocity $w_{i}$ described by Eq. (85). For example, this concerns particulate inclusions in an elastic environment that are small enough to diffuse through their surroundings, e.g., a polymeric network of significantly large mesh size. The dissipative contributions $\sim \zeta_{t r}^{(m n)}$ and $\sim \zeta_{l}^{(m n)}$, $(m n) \in\{(11),(12),(22)\}$, to $Z_{i j}^{(1) D}$ and $Z_{i j}^{(2) D}$ in Eqs. (79) and (80), respectively, imply that the components are not perfectly elastic. That is, they do not maintain statically deformed states, but rather relax the corresponding strains. Examples could be disentanglement processes in polymeric materials or filamentous networks. Corresponding relaxational processes render the systems viscoelastic and have to some extent already been investigated in Ref. [8]. Analogous conclusions apply to the relative translations between the two components, here entering through the corresponding contribution $\sim \zeta^{(\mathrm{rt})}$ via $z_{i}^{(\text {rel }) D}$ in Eq. (81).

\section{SUMMARY AND PERSPECTIVE}

In this work we have analyzed the macroscopic dynamics of elastic composite materials consisting of two components. As we have shown, two strain fields can be used for a nonlinear description. It turns out that two strain fields necessitate a two-fluid description with two velocity fields to describe the full dynamics. Alternatively to the two strain fields for the two subsystems, one can use the total strain field and the relative strain field. In addition, we have incorporated into our description the coupling of the two velocity fields to relative rotations, a feature not considered before. This effect leads to many reversible dynamic contributions also involving the two strain fields. For relative translations we concentrated on a linearized description. Large macroscopic relative translations would probably lead to a disintegration of the system into two pieces.

Our approach in the framework of generalized hydrodynamics is systematic in the low-frequency and longwavelength limit of moderate amplitudes, once the macroscopic variables have been defined. One of the strengths of the approach is that it allows one to work out the various mutual couplings among all these variables and their currents both in statics and dynamics. This statement is especially valid concerning the reversible dynamic parts. An obvious example is given by the ten reversible contributions to the dynamic strain equations that we have identified and that are based on the mean and relative velocities as further detailed in Appendix A.

Within the macroscopic theory, specific values of the listed coupling parameters cannot be provided. They depend on the material at hand and thus would need to be specified from experimental investigations, from coarse-graining microscopic models, or from numerical averaging of large-scale microscopic simulations. In this framework, it will be interesting to understand which microscopic features a substance needs to show so that the presently predicted macroscopic couplings emerge and can be validated.

Additional insight on the role and consequences of the derived contributions could be provided by corresponding macroscopic continuum simulations. For this purpose, it might be reasonable to start from a basic set of macroscopic variables and selected material parameters, excluding many of the listed contributions as far as possible. Then, successively, some of these contributions are added by varying the values of their associated material parameters. Their consequences are analyzed. In this way, an overview can be developed, particularly when the situations get more complex than in our investigated minimal examples, that is, when individual effects cannot be addressed in an isolated way any longer but when the coupling between many variables and spatial degrees of freedom becomes dominant. To facilitate corresponding computational evaluations, we have presented the effects of relative translations and rotations separately so that they can be added at a later stage. Moreover, we have included an overview on simplified sets of reversible contributions to the dynamic strain equations involving the mean and relative velocities.

In terms of further theoretical developments, the present investigation of two-component elastic systems with two velocity fields opens up a number of directions to pursue in the future. Naturally it will be an intriguing possibility to replace one of the solid components by magnetic particles or even by an already functionalized magnetic gel component, thus turning to the field of magnetorheological elastomers or even functionalized materials, one component of which already consists of a magnetic composite material itself. To this end we can make use of our previous work on two-fluid effects in magnetorheological fluids. The benefit of studying the effects of external magnetic fields in such a soft system will be to control shape and length changes by small to moderate magnetic fields. This approach can be used to build magnetic actuators [50-53]. Applying an oscillatory magnetic field to such a system with magnetic grains would also offer the possibility to induce oscillations in the nonmagnetic part of the system. In parallel, it seems worthwhile to study the electric analog to magnetorheological elastomers, namely, electrorheological elastomers. Such investigations may form a natural bridge in the context of improving the quality of current batteries.

Concerning the fields of chemical reactions and biological gels, another direction comes to mind. One could use a soft 
two-component system, for which one of the components supports an autocatalytic chemical reaction similar to the systems pioneered by Yoshida's group [54-56]. In this case the reaction couples to the shrinkage and expansion of the gel component hosting the chemical reaction in an oscillatory fashion. This time-dependent motion leading to deformations could then be transferred to the passive component. Clearly this concept could also be used in bioinspired soft matter systems sometimes called active media.

\section{ACKNOWLEDGMENTS}

Partial support of this work by the Deutsche Forschungsgemeinschaft through the Schwerpunktprogramm SPP 1681 "Feldgesteuerte Partikel-Matrix-Wechselwirkungen: Erzeugung, skalenübergreifende Modellierung und Anwendung magnetischer Hybridmaterialien" is gratefully acknowledged. Furthermore, A.M.M. thanks the Deutsche Forschungsgemeinschaft for support through Heisenberg Grant No. ME $3571 / 4-1$.

\section{APPENDIX A: REDUCING THE COMPLEXITY OF THE DYNAMIC STRAIN EQUATIONS}

The ten different velocities that enter the dynamic strain equations (39) and (40) are related to the mean velocity $v_{i}=\phi v_{i}^{(1)}+$ $[1-\phi] v_{i}^{(2)}$ and the relative velocity $w_{i}=v_{i}^{(1)}-v_{i}^{(2)}$ by

$$
\begin{gathered}
\tilde{v}_{i}^{(1)}=v_{i}+\alpha \beta_{5}^{(1)} w_{i}, \quad \tilde{v}_{i}^{(2)}=v_{i}+\alpha \beta_{5}^{(2)} w_{i}, \\
\hat{v}_{i}^{(11)}=v_{i}+\alpha \beta_{6}^{(1)} w_{i}, \quad \hat{v}_{i}^{(22)}=v_{i}+\alpha \beta_{6}^{(2)} w_{i}, \quad \hat{v}_{i}^{(12)}=\alpha \beta_{8}^{(1)} w_{i}, \quad \hat{v}_{i}^{(21)}=\alpha \beta_{8}^{(2)} w_{i}, \\
\breve{v}_{i}^{(11)}=v_{i}+\alpha \beta_{7}^{(1)} w_{i}, \quad \breve{v}_{i}^{(22)}=v_{i}+\alpha \beta_{7}^{(2)} w_{i}, \quad \breve{v}_{i}^{(12)}=\alpha \beta_{9}^{(1)} w_{i}, \quad \breve{v}_{i}^{(21)}=\alpha \beta_{9}^{(2)} w_{i} .
\end{gathered}
$$

The simplified Eqs. (41) and (42) are obtained by the special choices $\beta_{5}^{(1)}=\beta_{6}^{(1)}=\beta_{7}^{(1)}, \beta_{5}^{(2)}=\beta_{6}^{(2)}=\beta_{7}^{(2)}$, and $\beta_{8}^{(1)}=\beta_{8}^{(2)}=$ $\beta_{9}^{(1)}=\beta_{9}^{(2)}$ leading to the three velocities

$$
\bar{v}_{i}^{(1)}=v_{i}+\alpha \beta_{6}^{(1)} w_{i}, \quad \bar{v}_{i}^{(2)}=v_{i}+\alpha \beta_{6}^{(2)} w_{i}, \quad \bar{v}_{i}^{(3)}=\alpha \beta_{8}^{(1)} w_{i},
$$

depending on three phenomenological parameters.

The naive model, Eq. (43), is obtained by the additional choices $\beta_{8}^{(1)}=\beta_{8}^{(2)}=\beta_{9}^{(1)}=\beta_{9}^{(2)}=0$ leading to $\bar{v}_{i}^{(3)}=0$, and by $\alpha \beta_{6}^{(1)}=1-\phi$ and $\alpha \beta_{6}^{(2)}=-\phi$ leading to $\bar{v}_{i}^{(1)}=v_{i}^{(1)}$ and $\bar{v}_{i}^{(2)}=v_{i}^{(2)}$. In this model all phenomenological parameters $\beta_{5,6,7,8,9}^{(1,2)}$ are fixed.

The simplified model for $\dot{U}_{i j}^{\text {(tot) }}$ and $\dot{U}_{i j}^{(\text {rel })}$, Eqs. (45) and (46), with four phenomenological parameters, is obtained by the special choices $\beta_{n}^{(1)}=\beta_{n}^{(2)}$ for $n \in\{6,7\}$, while $\beta_{8}^{(1,2)}$ and $\beta_{9}^{(1,2)}$ are set to zero, leading to

$$
\begin{array}{cc}
\bar{v}_{i}^{(4)}=v_{i}+\alpha \beta_{6}^{(1)} w_{i}, & \bar{v}_{i}^{(6)}=v_{i}+\frac{1}{2} \alpha\left(\beta_{5}^{(1)}+\beta_{5}^{(2)}\right) w_{i}, \\
\bar{v}_{i}^{(5)}=v_{i}+\alpha \beta_{7}^{(1)} w_{i}, & \bar{v}_{i}^{(7)}=\frac{1}{2} \alpha\left(\beta_{5}^{(1)}-\beta_{5}^{(2)}\right) w_{i} .
\end{array}
$$

\section{APPENDIX B: SOME DETAILS OF TWO-FLUID HYDRODYNAMICS}

In this section we briefly sketch the structure of the previous two-fluid description involving two different densities $\rho^{(1,2)}$ and momenta $g_{i}^{(1,2)}$, where the focus is on genuine fluids in the absence of elasticity (cf. Refs. [14,15]). This implies the existence of two different velocities by the relations $g_{i}^{(1)}=\rho^{(1)} v_{i}^{(1)}$ and $g_{i}^{(2)}=\rho^{(2)} v_{i}^{(2)}$. The first law of thermodynamics in its differential form relates changes of the fluid variables to energy density and entropy density changes,

$$
d \varepsilon=T d \sigma+\mu^{(1)} d \rho^{(1)}+\mu^{(2)} d \rho^{(2)}+\boldsymbol{v}^{(1)} \cdot d \boldsymbol{g}^{(1)}+\boldsymbol{v}^{(2)} \cdot d \boldsymbol{g}^{(2)},
$$

defining the appropriate conjugate chemical potentials $\mu^{(1,2)}$. The conjugates to the momenta are the velocities according to the kinetic energy densities $\varepsilon_{1,2}^{\mathrm{kin}}=(1 / 2) v_{i}^{(1,2)} g_{i}^{(1,2)}$. We obtain the one-fluid limit by taking one of the densities as zero, or by setting $\rho^{(1)}=\rho^{(2)}$ and $v_{i}^{(1)}=v_{i}^{(2)}$.

This limit is facilitated (and the resulting dynamic equations are simplified) by switching to a different set of variables. In particular, we use the total momentum density $g_{i}=g_{i}^{(1)}+g_{i}^{(2)} \equiv \rho v_{i}$, thereby defining the mean velocity $v_{i}=(1 / \rho)\left(\rho^{(1)} v_{i}^{(1)}+\right.$ $\left.\rho^{(2)} v_{i}^{(2)}\right)$, which is also the conjugate to $g_{i}$, and the total density $\rho=\rho^{(1)}+\rho^{(2)}$, with the chemical potential $\mu$ as conjugate. Of course, one needs two further variables. For the density one can choose any rescaled linear combination of the two densities (other than the sum) and we use the concentration $\phi=\rho^{(1)} / \rho$. For the velocities a reasonable second variable is the velocity difference $w_{i}=v_{i}^{(1)}-v_{i}^{(2)}$. The one-fluid limit is then found from $\phi=0$ and $w_{i}=0$.

The Gibbs relation for this set of variables is given by Eq. (1), excluding the contributions of strain. Comparing with Eq. (B1) one finds for $m_{i}$, the conjugate of $w_{i}$, and for $\Pi$, the conjugate of $\phi$,

$$
\begin{aligned}
& \Pi=\left(\mu^{(1)}-\mu^{(2)}\right)+\boldsymbol{w} \cdot \boldsymbol{v}+\boldsymbol{w}^{2}(1-2 \phi) \quad \text { and } \quad \mu=\mu^{(1)} \phi+\mu^{(2)}(1-\phi)+\boldsymbol{w}^{2} \phi(1-\phi) \text {, } \\
& \text { or vice versa } \quad \mu^{(1)}=\mu+\rho^{-1} \rho^{(2)}\left(\Pi-\boldsymbol{w} \cdot \boldsymbol{v}_{1}\right) \quad \text { and } \quad \mu^{(2)}=\mu-\rho^{-1} \rho^{(1)}\left(\Pi+\boldsymbol{w} \cdot \boldsymbol{v}^{(2)}\right) \text {, }
\end{aligned}
$$




$$
\text { where } \quad \boldsymbol{v}=\phi \boldsymbol{v}^{(1)}+(1-\phi) \boldsymbol{v}^{(2)}=\rho^{-1}\left(\boldsymbol{g}^{(1)}+\boldsymbol{g}^{(2)}\right) \quad \text { and } \quad \boldsymbol{m}=\rho(1-\phi) \phi \boldsymbol{w}=\left(\rho^{(2)} \boldsymbol{g}^{(1)}-\rho^{(1)} \boldsymbol{g}^{(2)}\right) \rho^{-1} \text {. }
$$

Regarding the dynamical equations one has to take into account that the densities $\rho^{(1),(2)}$ are conserved individually and, in the simplest model, are transported by its velocity. The most general form is then

$$
\dot{\rho}^{(1)}+\nabla_{i}\left(\rho^{(1)} v_{i}^{(1)}+j_{i}^{(1)}\right)=0 \quad \text { and } \quad \dot{\rho}^{(2)}+\nabla_{i}\left(\rho^{(2)} v_{i}^{(2)}+j_{i}^{(2)}\right)=0
$$

with $j_{i}^{(2)}=-j_{i}^{(1)}$. Summing up these two equations, the phenomenological mass currents $j_{i}^{(1,2)}$ add up to zero, leading to $\dot{\rho}=$ $\nabla_{i} g_{i}$, Eq. (15), because the total mass current is equal to the total momentum density $\boldsymbol{g}=\rho \boldsymbol{v}$. For the concentration $\phi=\rho^{(1)} / \rho$ the dynamical equation (16) follows immediately from Eqs. (B5) as well.

However, it should be noted that the actual form, e.g., of the transport terms in a two-fluid description, are not $a$ priori fixed by any general rule. Rather, they depend on certain reversible phenomenological transport parameters, e.g., $\gamma$ in $j_{i}^{(1) R}$ of Eq. (37). In particular, for $\gamma=0$ (no transportlike contribution in $j_{i}^{(1) R}$ ) the effective transport velocities are $v_{i}^{(1,2)}$ for $\rho^{(1,2)}$, respectively, and $v_{i}^{(c)}=\left(\rho^{(2)} v_{i}^{(1)}+\rho^{(1)} v_{i}^{(2)}\right) / \rho$ for $\phi$ in Eq. (16), while for $\gamma=-1$ all three variables are convected by the mean velocity.

Similarly, the dynamics of the two momenta, convected with their appropriate velocities, takes the form

$$
\begin{aligned}
& \dot{g}_{i}^{(1)}+\nabla_{j} g_{i}^{(1)} v_{j}^{(1)}+\frac{\rho^{(1)} \rho^{(2)}}{\rho} X_{i}+\frac{\rho^{(1)}}{\rho} \nabla_{j} \sigma_{i j}-\frac{\rho^{(1)}}{\rho} w_{i} \nabla_{j} m_{j}-m_{j} \nabla_{j} v_{i}^{(1)}+v_{i}^{(c)} \nabla_{k} j_{k}^{(1)}=0, \\
& \dot{g}_{i}^{(2)}+\nabla_{j} g_{i}^{(2)} v_{j}^{(2)}-\frac{\rho^{(1)} \rho^{(2)}}{\rho} X_{i}+\frac{\rho^{(2)}}{\rho} \nabla_{j} \sigma_{i j}-\frac{\rho^{(2)}}{\rho} w_{i} \nabla_{j} m_{j}+m_{j} \nabla_{j} v_{i}^{(2)}-v_{i}^{(c)} \nabla_{k} j_{k}^{(1)}=0,
\end{aligned}
$$

with $v_{i}^{(c)}=\left(\rho^{(2)} v_{i}^{(1)}+\rho^{(1)} v_{i}^{(2)}\right) / \rho$. Summing up Eqs. (B6) and (B7), the required dynamic Eq. (17) for $g_{i}$ is obtained, because the momentum current density due to flow reads $\rho^{(1)} v_{i}^{(1)} v_{j}^{(1)}+\rho^{(2)} v_{i}^{(2)} v_{j}^{(2)}=g_{j} v_{i}+w_{j} m_{i}$. The stress tensor $\sigma_{i j}$ here comprises the isotropic pressure and the phenomenological contributions. The dynamic equation for the relative velocity, Eq. (18), follows from Eqs. (B6) and (B7) by subtraction [and using Eqs. (B5)], with $X_{i}$ the quasicurrent of $\dot{w}_{i}$ [including the $\nabla_{i}(\Pi / \rho)$ contribution] taking into account that $\beta_{2}=\beta_{4}=1 / 2$ and $\beta_{3}=1 / \rho_{1}-1 / \rho_{2}$ in $X_{i}^{R}$ of Eq. (35) is chosen.

[1] D. Myung, D. Waters, M. Wiseman, P.-E. Duhamel, J. Noolandi, C. N. Ta, and C. W. Frank, Polym. Adv. Technol. 19, 647 (2008).

[2] L. Zhang, S. P. Lake, V. H. Barocas, M. S. Shephard, and R. C. Picu, Soft Matter 9, 6398 (2013).

[3] A. S. Shahsavari and R. C. Picu, Phys. Rev. E 92, 012401 (2015).

[4] C. P. Brangwynne, F. C. MacKintosh, S. Kumar, N. A. Geisse, J. Talbot, L. Mahadevan, K. K. Parker, D. E. Ingber, and D. A. Weitz, J. Cell Biol. 173, 733 (2006).

[5] J. N. Coleman, U. Kan, and Y. K. Gun'ko, Adv. Mater. 18, 689 (2006).

[6] T. Goldacker, V. Abetz, R. Stadler, I. Erukhimovich, and L. Leibler, Nature (London) 398, 137 (1999).

[7] A. Böker, A. Knoll, H. Elbs, V. Abetz, A. H. E. Müller, and G. Krausch, Macromol. 35, 1319 (2002).

[8] A. M. Menzel, Phys. Rev. E 94, 023003 (2016).

[9] H. Wada and Y. Tanaka, Europhys. Lett. 87, 58001 (2009).

[10] J. M. van Doorn, L. Lageschaar, J. Sprakel, and J. van der Gucht, Phys. Rev. E 95, 042503 (2017).

[11] L. D. Landau and E. M. Lifshitz, Theory of Elasticity (Pergamon Press, Oxford, 1970).

[12] T. Gundermann and S. Odenbach, Smart Mater. Struct. 23, 105013 (2014).

[13] S. Odenbach, Arch. Appl. Mech. 86, 269 (2016).

[14] H. Pleiner and J. L. Harden, arXiv:cond-mat/0404134.
[15] H. Pleiner and J. L. Harden, in Slow Dynamics in Complex Systems: 3rd International Symposium on Slow Dynamics in Complex Systems, edited by M. Tokuyama and I. Oppenheim, AIP Conf. Proc. No. 708 (AIP, New York, 2006), p. 46.

[16] H. Pleiner, D. Svenšek, and H. R. Brand, Eur. Phys. J. E 36, 135 (2013).

[17] H. Pleiner, D. Svenšek, and H. R. Brand, Rheol. Acta 55, 857 (2016).

[18] H. Pleiner, D. Svenšek, T. Potisk, and H. R. Brand, Phys. Rev. E 101, 032601 (2020).

[19] I. M. Khalatnikov, Introduction to the Theory of Superfluidity (W. A. Benjamin, Reading, MA, 1965).

[20] P. C. Hohenberg and P. C. Martin, Ann. Phys. (NY) 34, 291 (1965).

[21] R. Graham, Phys. Rev. Lett. 33, 1431 (1974).

[22] R. Graham and H. Pleiner, Phys. Rev. Lett. 34, 792 (1975).

[23] M. Liu, Phys. Rev. B 13, 4174 (1976).

[24] R. Graham and H. Pleiner, J. Phys. C 9, 279 (1976).

[25] H. R. Brand, M. Dörfle, and R. Graham, Ann. Phys. 119, 434 (1979).

[26] P. G. de Gennes, in Liquid Crystals of One- and TwoDimensional Order, edited by W. Helfrich and G. Heppke (Springer, Berlin, 1980), pp. 231-237.

[27] S. Bohlius, H. R. Brand, and H. Pleiner, Phys. Rev. E 70, 061411 (2004).

[28] T. Potisk, H. Pleiner, and H. R. Brand, Eur. Phys. J. E 42, 35 (2019). 
[29] A. M. Menzel, H. Pleiner, and H. R. Brand, J. Chem. Phys. 126, 234901 (2007).

[30] A. M. Menzel, H. Pleiner, and H. R. Brand, J. Appl. Phys. 105, 013503 (2009).

[31] A. M. Menzel, H. Pleiner, and H. R. Brand, Eur. Phys. J. E 30, 371 (2009).

[32] H. Temmen, H. Pleiner, M. Liu, and H. R. Brand, Phys. Rev. Lett. 84, 3228 (2000).

[33] H. Temmen, H. Pleiner, M. Liu, and H. R. Brand, Phys. Rev. Lett. 86, 745 (2001).

[34] H. Pleiner, M. Liu, and H. R. Brand, Rheol. Acta 39, 560 (2000).

[35] M. Grmela, Phys. Lett. A 296, 97 (2002).

[36] H. Pleiner, M. Liu, and H. R. Brand, Rheol. Acta 41, 375 (2002).

[37] H. Pleiner, M. Liu, and H. R. Brand, Rheol. Acta 43, 502 (2004).

[38] H. Pleiner, M. Liu, and H. R. Brand, in Modeling of Soft Matter, IMA Volumes in Mathematics and its Applications, Vol. 141: Flu$i d s$, edited by M.-C. T. Calderer and E. M. Terentjev (Springer, New York, 2005), p. 99.

[39] O. Müller, M. Liu, H. Pleiner, and H. R. Brand, Phys. Rev. E 93, 023113 (2016).

[40] O. Müller, M. Liu, H. Pleiner, and H. R. Brand, Phys. Rev. E 93, 023114 (2016).

[41] H. B. Callen, Thermodynamics and an Introduction to Thermostatics (Wiley, New York, 1985).

[42] H. Pleiner and H. R. Brand, in Pattern Formation in Liquid Crystals, edited by A. Buka and L. Kramer (Springer, New York, 1996).
[43] C. Pantea, I. Stroe, H. Ledbetter, J. B. Betts, Y. Zhao, L. L. Daemen, H. Cynn, and A. Migliori, Phys. Rev. B 80, 024112 (2009).

[44] S. Huang, G. Pessot, P. Cremer, R. Weeber, C. Holm, J. Nowak, S. Odenbach, A. M. Menzel, and G. K. Auernhammer, Soft Matter 12, 228 (2016).

[45] M. Puljiz and A. M. Menzel, Phys. Rev. E 95, 053002 (2017).

[46] M. Puljiz and A. M. Menzel, Phys. Rev. E 99, 053002 (2019).

[47] S. Goh, A. M. Menzel, and H. Löwen, Phys. Chem. Chem. Phys. 20, 15037 (2018).

[48] S. Palagi, A. G. Mark, S. Yik Reigh, K. Melde, T. Qiu, H. Zeng, C. Parmeggiani, D. Martella, A. Sanchez-Castillo, N. Kapernaum, F. Giesselmann, D. S. Wiersma, E. Lauga, and P. Fischer, Nat. Mater. 15, 647 (2016).

[49] S. Jahanshahi, C. Lozano, B. Liebchen, H. Löwen, and C. Bechinger, Commun. Phys. 3, 127 (2020).

[50] R. Fuhrer, E. K. Athanassiou, N. A. Luechinger, and W. J. Stark, Small 5, 383 (2009).

[51] H. Böse, R. Rabindranath, and J. Ehrlich, J. Intell. Mater. Syst. Struct. 23, 989 (2012).

[52] J. Maas and D. Uhlenbusch, Smart Mater. Struct. 25, 104002 (2016).

[53] L. Fischer and A. M. Menzel, Phys. Rev. Res. 2, 023383 (2020).

[54] R. Yoshida, T. Takahashi, T. Yamaguchi, and H. Ichijo, J. Am. Chem. Soc. 118, 5134 (1996).

[55] R. Yoshida, T. Takahashi, T. Yamaguchi, and H. Ichijo, Adv. Mater. 9, 175 (1997).

[56] R. Yoshida, M. Tanaka, S. Onodera, T. Yamaguchi, and E. Kokufuta, J. Phys. Chem. A 104, 7549 (2000). 